\title{
Brain Dynamics of Aging: Multiscale Variability of EEG Signals at Rest and during an Auditory Oddball Task ${ }^{1,2,3}$
}

\author{
(D) Rita Sleimen-Malkoun, ${ }^{1,2, *}$ Dionysios Perdikis, ${ }^{1,3, *}$ Viktor Müller, ${ }^{3}$ Jean-Luc Blanc, ${ }^{1,4}$ Raoul Huys, ${ }^{1,4}$ \\ Jean-Jacques Temprado, ${ }^{2}$ and Viktor K. Jirsa ${ }^{1,4}$
}

DOI:http://dx.doi.org/10.1523/ENEURO.0067-14.2015

\begin{abstract}
${ }^{1}$ Aix-Marseille Université, Inserm, Institut de Neurosciences des Systèmes UMR_S 1106, 13385, Marseille, France, ${ }^{2}$ Aix-Marseille Université, CNRS, Institut des Sciences du Mouvement UMR 7287, 13288, Marseille, France, ${ }^{3}$ Max Planck Institute for Human Development, Center for Lifespan Psychology, 14195, Berlin, Germany, and ${ }^{4}$ CNRS, 13402, Marseille, France
\end{abstract}

\begin{abstract}
The present work focused on the study of fluctuations of cortical activity across time scales in young and older healthy adults. The main objective was to offer a comprehensive characterization of the changes of brain (cortical) signal variability during aging, and to make the link with known underlying structural, neurophysiological, and functional modifications, as well as aging theories. We analyzed electroencephalogram (EEG) data of young and elderly adults, which were collected at resting state and during an auditory oddball task. We used a wide battery of metrics that typically are separately applied in the literature, and we compared them with more specific ones that address their limits. Our procedure aimed to overcome some of the methodological limitations of earlier studies and verify whether previous findings can be reproduced and extended to different experimental conditions. In both rest and task conditions, our results mainly revealed that EEG signals presented systematic age-related changes that were time-scale-dependent with regard to the structure of fluctuations (complexity) but not with regard to their magnitude. Namely, compared with young adults, the cortical fluctuations of the elderly were more complex at shorter time scales, but less complex at longer scales, although always showing a lower variance. Additionally, the elderly showed signs of spatial, as well as between, experimental conditions dedifferentiation. By integrating these so far isolated findings across time scales, metrics, and conditions, the present study offers an overview of age-related changes in the fluctuation electrocortical activity while making the link with underlying brain dynamics.
\end{abstract}

Key words: aging; brain dynamics; brain signal variability; complexity; EEG; multiscale entropy

\section{Significance Statement}

Recently, the study of brain signal fluctuations is widely put forward as a promising entry point to characterize brain dynamics in health and disease. Although interesting results have been reported regarding how variability of brain activations can serve as an indicator of performance and adaptability in elderly, many uncertainties and controversies remain with regard to the comparability, reproducibility, and generality of the described findings, as well as the ensuing interpretations. Following a systematic investigation of these issues by using a large set of metrics and different experimental conditions, our results draw an overview of age-related changes of the magnitude and structure of brain fluctuations, which integrate well with known structural and functional alterations, as well as the main aging theories. 


\section{Introduction}

The view that variability in brain activity serves a functional role is gaining increasing support (Ghosh et al., 2008; Deco et al., 2009, 2011, 2013; Garrett et al., 2011; Hong and Rebec, 2012). The characteristics of brain signal fluctuations are considered to capture the underlying complex interactions between neuronal structures and ensembles.

At rest, the brain displays a complex though spatiotemporally structured dynamics, where brain states known as resting-state networks are intermittently activated. These states are considered to be functionally meaningful because several of them have been known from task paradigms (Deco et al., 2013). As underlying mechanisms, within deterministic frameworks, heteroclinic cycles have been proposed to generate sequential transitions from one unstable equilibrium point (saddle) to another. Other deterministic approaches soften the requirement of unstable states and require linked attractive subspaces (Huys et al., 2014). These approaches are subject to noise, which seems to be pervasive at different levels of the CNS (Faisal et al., 2008). However, they do not necessarily require the latter as a generative element as do those considering that the continually fluctuating background activity, random or not, drives the multistable system through a cascade of epochs of invariant, but distinct, coordinated network activities (Hansen et al., 2015). Mclntosh et al. (2010) argued that noise is linked to an increased number of functional network configurations that can be occupied in stochastic systems. This suggests that maturational changes in brain noise represent an enhancement of the functional network potential, the brain's dynamic repertoire (Ghosh et al., 2008). Conversely, the natural process of aging, as well as disease, has been associated with an evolution toward poorer dynamics, more local interactions, and more regular fluctuations in brain and behavior (for review, see Garrett et al., 2013b; Sleimen-Malkoun et al., 2014).

In the ergodic theory framework, entropy has been theoretically demonstrated to be an nonredundant measure of dynamical systems (Adler and Weiss, 1967; Ornstein and Weiss, 1991). In empirical data, neurobehavioral variability is characterized through the magnitude

Received December 1, 2014; accepted March 16, 2015; First published May 11, 2015.

${ }^{1}$ The authors report no financial conflicts of interest.

${ }^{2}$ Author contributions: R.S.-M., D.P., V.M., and V.K.J. designed research; R.S.-M., D.P., V.M., and V.K.J. performed research; R.S.-M., D.P., and J.L.-B. analyzed data; R.S.-M., D.P., V.M., R.H., J.-L.B., J.-J.T. and V.K.J. wrote the paper.

${ }^{3}$ This work was supported by the Aix-Marseille Université foundation A*Midex-CoordAge project, and the Max Planck Society.

${ }^{*}$ R.S.-M. and D.P. contributed equally to this work.

Correspondence should be addressed to Viktor Jirsa, Aix-Marseille Université, Inserm, Institut de Neurosciences des Systèmes UMR_S 1106, 13385, Marseille, France. E-mail: viktor.jirsa@univ-amu.fr.

DOI:http://dx.doi.org/10.1523/ENEURO.0067-14.2015

Copyright (C) 2015 Sleimen-Malkoun et al.

This is an open-access article distributed under the terms of the Creative Commons Attribution 4.0 International, which permits unrestricted use, distribution and reproduction in any medium provided that the original work is properly attributed. (variance-derived measures) and the time structure (longrange correlations and entropy-derived metrics; Bravi, et al., 2011) of fluctuations. The main operational principle is that the healthy system exhibits complex fluctuations somewhere at a sweet spot between randomness and regularity. Such resonance-like phenomena are known as stochastic resonance and have been observed in biological systems including brain networks (Gammaitoni et al., 1998; Deco et al., 2009; McDonnell and Abbott, 2009; McDonnell and Ward, 2011). Nevertheless, most of the widely used measures cannot distinguish between deterministic and stochastic components of the dynamics. Entropy measures, for instance, are relevant for comparisons between different conditions (e.g., resting vs task) or systems (e.g., young vs old), assuming conventionally that more entropy corresponds to more complexity (Feldman and Crutchfield, 1998). Sensu stricto, this latter assumption is not always correct, at least not with single-scale measures (Costa et al., 2002, 2005).

In fMRI studies, variance-based measures (Grady and Garrett, 2014), as well as entropy measures (Liu et al., 2013; Sokunbi, 2014), have been shown to be relevant to characterize and understand the dynamics of the aging brain. In this context, multiscale analyses have also been used (Yang et al., 2013; Smith et al., 2014), although, their contribution is restricted due to the limited range of functionally meaningful scales that can be covered. Such measures are of more interest in signals with higher time resolution, as electroencephalogram (EEG) and MEG recordings, where time-scale dependence of aging effects can be revealed (Mclntosh et al., 2014). Nevertheless, notwithstanding a number of converging findings showing that aging does affect the variability of brain activity, no final conclusions can be made yet concerning the nature of such changes or their link with functional and adaptive capabilities. The present study makes a helpful step in this direction by offering a consistent and coherent characterization of EEG signals in young and older adults through a multiplicity of metrics applied to both resting and task conditions. Specifically, it investigates the following: (1) the type of information that can (or cannot) be captured by the (univariate) metrics that are conventionally used to characterize brain signals, (2) the distinction between multiscale changes in the magnitude of fluctuations and their structure in time, (3) the correspondences between different classes of metrics with regard to age-related modifications in brain activity, (4) the comparability between aging effects on resting and task-evoked brain fluctuations, and (5) the extent to which changes in brain fluctuations can be linked to structural and functional changes occurring in the aging brain.

\section{Methods}

\section{Participants}

Participants were recruited through announcements at schools in Saarland and at the Saarland University. They received a compensation of 7.5 Euro per hour. All the participants were right-handed, had no reported history of head or neurological disorders, and none were on medication. The studied sample consisted of 31 young 
$(\mathrm{Y}$; mean age $=22.7, \mathrm{SD}=1.6$, age range $=18.8-25.1$ years, 14 females), and 28 old adults $(\mathrm{O}$; mean age $=$ $67.8, \mathrm{SD}=3.0$, age range $=63.9-74.5$ years, 14 females). Participants of all ages were able to sustain their attention for the entire duration of the experiment, and they all underwent a psychological and audiological assessment prior to their enrollment. The used protocol was in accordance with the regulation of the local ethics committee. All participants volunteered for this experiment and gave their written informed consent prior to their inclusion in the study.

\section{Procedure}

The EEG measurement began with a 3 min resting-state recording (1.5 min with eyes closed, and $1.5 \mathrm{~min}$ with eyes open) and was followed by the auditory oddball task. During the task, participants were seated comfortably on a chair in an electrically shielded room, with their eyes closed. They heard two different tone beeps: a frequent $1000 \mathrm{~Hz}$ tone as a standard stimulus and a rare $800 \mathrm{~Hz}$ tone as a deviant stimulus. The standard and deviant stimuli were presented binaurally (with a probability of 0.8 and 0.2 for standard and deviant, respectively) through headphones (Sony DJMDR-V300) at $70 \mathrm{~dB}$ SPL with duration of $70 \mathrm{~ms}$ (including $10 \mathrm{~ms}$ rise and fall time). Stimuli were generated with the software Audacity 1.2.4. The interstimulus interval ranged from 1200 to $1500 \mathrm{~ms}$. There were two different experimental conditions: passive listening (unattended) and active counting (attended). In the first condition, participants merely listened to the tone beeps without any response, whereas in the second condition, they had to attend to stimuli and to count the deviant tones. After the session, they were asked to report their counting results. Each experimental condition contained 152 standard tones and 38 deviant tones presented in a pseudorandom order fixed for all participants. The order of the conditions was always the same, with the active counting condition following the passive listening condition. For this study, we considered three conditions, all with eyes closed: resting state $(\mathrm{R})$, auditory oddball task without counting (OnC), and auditory oddball with counting (OC). The condition of resting state with eyes open was not included because it differed largely in its frequency content compared to all other conditions, which interfered with tasks contrasts. Instead, we focused on studying differences under comparable conditions along the axis of increasing attentional and task demands.

\section{EEG recordings and preprocessing}

The electroencephalogram (EEG) was recorded from $58 \mathrm{Ag} / \mathrm{AgCl}$ electrodes using an elastic cap (Electrocap), with a sampling rate of $500 \mathrm{~Hz}$ in a frequency band ranging from 0.5 to $100 \mathrm{~Hz}$. The left mastoid was used as a reference and the right mastoid was recorded as an active channel. The data were re-referenced offline to an average of the left and right mastoids for further analysis. The electrodes were placed according to the international $10-10$ system. Vertical and horizontal electrooculogram was recorded for control of eye blinks and eye movements. Eye movement correction
Table 1 Mean, SD, and minimum and maximum numbers of EEG segments per group and condition included in the analysis

\begin{tabular}{llllll}
\hline \multirow{4}{*}{ Young } & Rest & Mean & SD & Min & Max \\
& OnC & 2.8 & 0.6 & 5 & 8 \\
\multirow{4}{*}{ Old } & OC & 23.9 & 2.0 & 15 & 25 \\
& Rest & 7.4 & 3.1 & 11 & 25 \\
& OnC & 22.3 & 1.1 & 4 & 8 \\
& OC & 21.6 & 3.4 & 12 & 25 \\
& & & & 15 & 25 \\
\hline
\end{tabular}

was accomplished by independent component analysis (Vigario, 1977). Thereafter, artifacts from head and body movements were rejected by visual inspection. Finally, data were downsampled to a sampling rate of $250 \mathrm{~Hz}$, segmented in artifact free $10 \mathrm{~s}$ segments (i.e., comprising $N_{t}=2500$ data points each), and mean centered within segments before further analysis. Accordingly, we insured to have continuous time series of equal length for all three experimental conditions, on which multiscale analyses can be reliably applied. For the two task conditions, segments corresponded to time intervals containing a comparable number of stimuli (7-8). Table 1 shows the statistics of the resulting number of segments included in the analysis for each condition and group.

\section{Metrics}

Multiple metrics were applied to all data segments using MATLAB (MathWorks) or Python scripts for all calculations. We computed: the power spectrum, the spectral degrees of freedom, the detrended fluctuation analysis, the variogram, and several measures related to multiscale entropy. In general, all of these metrics relate in some way to the autocorrelation properties of the signals. However, it should be noted that neither a straightforward relationship amongst metrics, nor a direct correspondence between time scales and frequencies exist. On the one hand, the entropic measures and detrended fluctuation analysis capture nonlinear correlations in addition to linear ones, but it is not the case for the variogram and the power spectrum. On the other hand, the detrending and the coarse graining procedures (for entropic measures) transform the data in ways that make such direct correspondence impossible. In the following sections, we present the different metrics.

\section{Power spectrum}

For the calculation of the power spectrum $(P)$, we applied a Hanning window of $N_{t}=2500$ points to each data segment. Then, after padding with trailing zeros, a 4096 point Fast Fourier Transform (using the MATLAB function fft.m) resulted in the complex signal in the frequency domain $X(k)=\sum_{j=1}^{N_{p}}\left(x(j) e^{\left(-2 \pi / N_{p}\right)(j-1)(k-1)}\right)$, where $x$ is the signal in the time domain, $N_{p}=4096$ and indices $j$ and $k$ run through points in the time and frequency domain, respectively. Then, the power spectrum was calculated for positive frequencies as $P(k)=X(k) X(k)^{*}$, where the operator * signifies the conjugate complex number. 


\section{Degrees of freedom}

Spectral degrees of freedom $(D o F)$ is a statistic that evaluates the uniformity of spectral density (Vaillancourt and Newell, 2003). It is calculated as $\operatorname{DoF}=\left(\sum_{k}^{N_{f}} P(k)\right)^{2} / 1 / N_{f} \sum_{k}^{N_{f}} P(k)^{2}$, where $P$ and $k$ are as above and $N_{f}$ is the number of positive frequencies. DoF ranges from $1 / N_{f}$ for a single peak spectral density to 1 for a completely flat one, i.e., for white noise.

\section{Detrended fluctuation analysis and generalized Hurst exponent}

Detrended fluctuation analysis (DFA) was introduced (Peng et al., 1994) in order to extent Hurst's (1951) Rescaled Range Analysis for the evaluation of long-range time correlations in nonstationary signals. Its suitability for nonstationary signals has been questioned recently (Bryce and Sprague, 2012). However, it is widely used in different domains and has found many applications in biology (Hardstone et al., 2012 provides details for applications in EEG). We calculated DFA along the following steps:

1. We calculated the cumulative sum of each segment's time series after removal of its mean: $y$ $(j)=\sum_{1}^{j}\left(x(j)-\sum_{k}^{N_{t}} x(j) / N_{t}\right)$, where all symbols follow the above presented notation.

2. For a particular time scale $T(s)$, with scale $s=$ 4. . .50, and $T=16$. .200 $\mathrm{ms}$ in steps of $4 \mathrm{~ms}$, we segmented the time series into adjacent (nonoverlapping) windows $y_{w s}$ of a length of $N_{w}(s)$ samples. Thus, the number of windows $W(s)$ ranged as $W=$ 625 . . 50, and the number of samples per window as $N_{w}=4$. .50, respectively.

3. For each scale $s$ we calculated the average fluctuation across all windows as the average rootmean-square error of a polynomial fit of second order (i.e., it corresponds to removal of linear trends):

4.

$$
F(s)=\left(\sum_{s}^{w(s)} \sqrt{1 / N_{w}(s)\left(\sum_{m}^{N_{w}(s)}\left(y_{w s}(m)-\left(a_{2} m^{2}+a_{1} m+a_{0}\right)\right)^{2}\right)}\right)
$$

$M(s)$, where $a_{0-2}$ are the coefficients of the polynomial fit, and $m$ is the index of all samples within a window. We used the MATLAB functions polyfit.m and polyval.m for the calculations of the polynomial coefficients and fitting, respectively.

5. Fluctuations were plotted against time scales in a In $T(s)$ - In $F(s)$ plot and a generalization of the Hurst exponent, $H$, was calculated as the slope of the linear fit (using polyfit in MATLAB) of the resulting curve for time scales $T$ in the range $24-124 \mathrm{msec}$. This range was chosen after visual inspection for linear scaling of randomly chosen data segments as well as of the groups' mean curves for each condition. Finally, we compared both In $F(s)$ and $H$ across groups and conditions.

$H$ is indicative of the autocorrelation structure of a signal as follows: (a) for $0<H<0.5$, negative correlation (anticorrelation), (b) for $H \approx 0.5$, lack of any correlation, i.e., white noise, (c) for $0.5<H<1$, positive correlation, (d) for $H \approx 1,1 / f$ or pink noise, (e) for $1<H<2$, nonstationarity, (f) for $H \approx 1.5$, brown noise. The Hurst exponent is equal to $H$ for $H<1$ and to $H-1$ for $H>1$ (Hardstone et al., 2012).

\section{Variogram}

The variogram $(V)$ is an alternative way to evaluate how the magnitude of variability of a signal varies for different time scales (Cressie, 1993). However, until present its use has been limited in neurosciences (Conte et al., 2009). It has the advantage over variance in that it can be calculated for stochastic processes for which the mean is either undefined, i.e., when the related probability distribution function decays according to a power law with an exponent less than or equal to 1 , or when it is hard to empirically observe, i.e., in the cases of a very large autocorrelation time. It was calculated as: $V(s)=1 / N_{s}$ $\left(\sum_{s}^{N_{s}}(x(j)-x(j+s))^{2}\right)$, where $N_{s}$ is the number of distinct páirs of time points $x(j)$ and $x(j+s)$ of a distance of $s$ samples, in the range $s=1 \ldots .50$, which corresponds to time scales $T(s)$ in the range of $4-200$ msec. Finally, we compared $\ln V(s)$ among groups and conditions.

\section{Multiscale entropy measures}

We calculated multiscale entropy using two different estimators: sample entropy (SampEn; Richman and Moorman, 2000), giving multiscale sample entropy (MSE), and Lempel-Ziv complexity (LZ; Lempel and Ziv, 1976), yielding multiscale Lempel-Ziv entropy (MLZ). To improve the interpretability of our results, we also estimated a normalized version of each, i.e., MSEn and MLZn (see below).

MSE was introduced by Costa et al. $(2002,2005)$ to evaluate the complexity of physiobiological signals, such as heart rate, i.e., the degree to which long-range correlations exist in such signals. The MSE algorithm combines the calculation of SampEn with a coarse graining procedure, acting similar, albeit not identical, to a low-pass filter, thereby precluding a one-to-one comparison between time scales and frequency content of the signal. SampEn is an improved version of the approximate entropy algorithm (Pincus, 1991), which has been designed to approximate the so-called Kolmogorov-Sinai entropy of dynamical systems (that quantifies the global temporal organization of time series and provides a meaningful index for discriminating between various dynamic systems), or the metric entropy or mean entropy rate of stochastic processes (that is, the rate with which such processes create new information), for time series of relatively short length, as it is usually the case in biology. In short, we calculated MSE along the following steps:

1. For a particular time scale $T(s)$, with scale $s=1 \ldots .50$, and $T=4 \ldots 200 \mathrm{~ms}$ in steps of $4 \mathrm{~ms}$, we segmented the time series $x(j)$ into adjacent (nonoverlapping) windows $y_{w s}$ of a length of $N_{w}(s)$ samples. Thus the number of windows $W(s)$ ranged as $W=2500 \ldots 50$, and the number of samples per window as $N_{w}=$ 1 . . .50, respectively.

2. We averaged all points within each window $y_{w s}$ to generate new time series $z_{w s}=1 / N_{w}(s) \sum_{j=1}^{N_{w}(s)} y_{w s}(j)$ for each scale $s$.

3. Then, SampEn was calculated for each of the $z_{w s}$ 
time series, resulting in a SampEn value for each scale, as $\operatorname{MSE}(s)=-\ln (N(m+1) / N(m))$, where $N$ $(m)$ is the number of all possible sequences of $m$ points in $z_{w s}$ that are closer to each other than a distance $r$, i.e., where $\left(\left|z_{w s}(i)-z_{w s}(j)\right|<r\right) \bigcap$ $\left(\left|z_{w s}(i+1)-z_{w s}(j+1)\right|<r\right) \bigcap$. . $\left(\mid z_{w s}(i+\right.$ $m-1)-z_{w s}(j+m-1) \mid<r$ ) and $i<j$ (no self-matches are counted). Thus, SampEn evaluates the percentage of similar sequences of $m$ points that are still similar (in terms of distance) when the next point, i.e., the $m+1$, is added to the sequence. In all our calculations we set $m=2$ and $r$ as $50 \%$ of the SD of the original signal $x(j)$, i.e., at scale 1.

However, the SampEn algorithm has not been analytically proven to converge toward metric entropy and requires a preliminary setting of the parameter $m$ that could lead to an underestimation if set inappropriately. We therefore also tested the $L Z$ complexity, which is an adaptive entropy estimator. In addition to being parameterfree, it was shown to be reliable even for short sequences of a few hundreds of symbols (Lesne et al., 2009). We used the same procedure as described above, but at step 3 , we calculated $L Z$ instead of SampEn. In the $L Z$ compression algorithm, a symbolic sequence of length $N_{s}$ is parsed recursively into words, considering as a new word the shortest one that has not yet been encountered. For instance, in a binary example the sequence 100110111001010001011 . is parsed according to 1.0 . $01.10 .11 .100 .101 .00 .010 .11 \ldots$. One then computes $L Z=N_{w}\left(1+\log _{k} N_{w}\right) / N_{s}$, where $N_{w}$ is the number of words used and $k$ is the number of symbols in the "alphabet". Under the assumption that the source is stationary and ergodic (assumptions that apply to the SampEn estimator as well), Lempel-Ziv theorems ensure that LZ coincides with the entropy rate up to a factor logk with $\lim _{N \rightarrow \infty} L Z=h /$ logk, where $h$ corresponds to metric entropy.

We used an equiquantization procedure (Hlaváčková et al., 2007) to convert signals into symbolic sequences by partitioning them into four bins $(k=4)$. The bin size was inversely proportional to the distribution of the amplitude values of EEG, such that the number of values was the same in all bins.

MSE curves have been shown to be highly influenced by the effect of the coarse graining procedure on the SD at each scale (Nikulin and Brismar, 2004). Therefore, we also calculated the SD across scales $S D(s)$ (i.e., after coarse graining) as well as MSEn(s), for which we set a different threshold $r(s)$ for each scale that was equal to $50 \%$ of SD(s) (i.e., relative to the SD of the coarse grained signal $Z_{w s}$ ). This normalization was also applied to MLZ by applying at each scale a new grid, adjusted to the variance of the coarse-grained signal.

\section{Partial Least Squares statistical analysis}

We used "contrast" or "non-rotated task partial least squares" (PLS; as implemented in MATLAB by Mclntosh and Lobaugh, 2004; Krishnan et al., 2011 provides updated information) to test the main effects of groups and conditions differences. In a nutshell, contrast task PLS is a multivariate statistical method that is suitable for testing hypotheses about spatial and/or time distributed signal changes by combining information across the different signal dimensions (in our case, channels and time scales or frequencies). PLS addresses both the problem of multiple comparisons for statistical significance and of that of elementwise reliability via a permutation test and a bootstrap resampling test, respectively. A task PLS analysis with $N_{g}$ groups and $N_{c}$ conditions starts with a data matrix for each group and a contrast matrix of maximally $N_{g} \times N_{c}-1$ (as many as the degrees of freedom) orthonormal contrasts that represent the hypotheses to be tested. The rows of each data matrix contain a metric's data points or elements of participants within conditions, which in our case were a metric's values for all channel and time scale or frequency combinations. From those two matrices, a covariance matrix is calculated that contains the covariance of each orthonormal contrast with each element across participants. This matrix is subjected to singular value decomposition (SVD) resulting in three matrices: (1) the orthonormal matrix of the saliences of the contrasts (as determined by the initial contrast matrix) i.e., it contains the task (or design) latent variables that describe the relations among the conditions and groups of our design; (2) the orthonormal matrix of element saliences that are proportional to the covariance of each metrics' element with each one of the task contrasts, i.e., it describes the so-called brain latent variables; and (3) the diagonal matrix of singular values that are indicative of the variance explained by each contrast. Then, a permutation test on the singular values, with resampling of the initial data matrices, results in a $p$ value for each contrast tested. Finally, a bootstrap test with resampling of the initial data matrices, with replacement within conditions and groups, results in statistical reliability estimations of each element of both the task and the brain latent variables within a chosen level of confidence. Thus, the bootstrap test controls for the robustness of the results among participants. For the task latent variables, we plotted intervals of $95 \%$ confidence. Conditions with nonoverlapping intervals are robustly distinguished by the respective contrast. For the brain latent variables, we calculated bootstrap ratios by dividing each element with its SE as calculated by the corresponding bootstrap sample distribution. Bootstrap ratios $>2.5758$ approximate the $99^{\text {th }}$ two-tailed percentile for a particular element. Regarding the statistical table (Table 2), we calculated the AgrestiCoull 95\% confidence intervals for the $p$ value of all permutation tests, assuming a binomial distribution for the probability that a permutation sample will lead to a larger eigenvalue than the observed one (Brown et al., 2001), whereas for the bootstrap tests we direct the reader to the corresponding figures, where the confidence intervals of the task latent variables and the bootstrap ratios of the brain latent variables are depicted.

In our design, we had two groups (i.e., $N_{g}=2$ ), namely $Y$ and old $O$ participants, and three conditions $\left(N_{c}=3\right)$, i.e., $\mathrm{R}, \mathrm{OnC}$, and $\mathrm{OC}$ as explained above. We tested two orthogonal contrasts. The weights for the first one before 
Table 2 Statistical table

\begin{tabular}{|c|c|c|c|c|}
\hline \multirow{11}{*}{$\begin{array}{l}\text { Effect } \\
\text { Group main effect }\end{array}$} & Metric & Data structure & Type of test & Confidence intervals \\
\hline & & & $\begin{array}{l}\text { permutation } \\
\text { bootstrap }\end{array}$ & $\begin{array}{l}{[-0.0008,0.0046]} \\
\text { Figure } 5 \text { for Task LV confidence intervals and } \\
\text { Figure } 6 \text { for the Brain LV bootstrap ratios }\end{array}$ \\
\hline & & & bootstrap & $\begin{array}{l}\text { Figure } 5 \text { for Task LV confidence intervals and } \\
\text { Figure } 6 \text { for the Brain LV bootstrap ratios }\end{array}$ \\
\hline & SD & & permutation & {$[-0.0008,0.0046]$} \\
\hline & $\ln (F)$ & & $\begin{array}{l}\text { permutation } \\
\text { bootstrap }\end{array}$ & $\begin{array}{l}{[-0.0008,0.0046]} \\
\text { Figure } 5 \text { for Task LV confidence intervals and } \\
\text { Figure } 6 \text { for the Brain LV bootstrap ratios }\end{array}$ \\
\hline & DoF & & $\begin{array}{l}\text { permutation } \\
\text { bootstrap }\end{array}$ & $\begin{array}{l}{[-0.0008,0.0046]} \\
\text { Figure } 5 \text { for Task LV confidence intervals and } \\
\text { Figure } 7 \text { for the Brain LV bootstrap ratios }\end{array}$ \\
\hline & MSEn & & $\begin{array}{l}\text { permutation } \\
\text { bootstrap }\end{array}$ & $\begin{array}{l}{[-0.0008,0.0046]} \\
\text { Figure } 5 \text { for Task LV confidence intervals and } \\
\text { Figure } 8 \text { for the Brain LV bootstrap ratios }\end{array}$ \\
\hline & MLZ & & permutation & {$[-0.0008,0.0046]$} \\
\hline & & & bootstrap & $\begin{array}{l}\text { Figure } 5 \text { for Task LV confidence intervals and } \\
\text { Figure } 8 \text { for the Brain LV bootstrap ratios }\end{array}$ \\
\hline & MLZn & & permutation & {$[-0.0008,0.0046]$} \\
\hline & & & bootstrap & $\begin{array}{l}\text { Figure } 5 \text { for Task LV confidence intervals and } \\
\text { Figure } 8 \text { for the Brain LV bootstrap ratios }\end{array}$ \\
\hline \multirow{11}{*}{$\begin{array}{l}\text { Condition main } \\
\text { effect }\end{array}$} & $P$ & & permutation & {$[-0.0008,0.0046]$} \\
\hline & & & bootstrap & $\begin{array}{l}\text { Figure } 9 \text { for Task LV confidence intervals and } \\
\text { Figure } 10 \text { for the Brain LV bootstrap ratios }\end{array}$ \\
\hline & & & bootstrap & $\begin{array}{l}\text { Figure } 9 \text { for Task LV confidence intervals and } \\
\text { Figure } 10 \text { for the Brain LV bootstrap ratios }\end{array}$ \\
\hline & MSE & & permutation & {$[-0.0008,0.0046]$} \\
\hline & & & bootstrap & $\begin{array}{l}\text { Figure } 9 \text { for Task LV confidence intervals and } \\
\text { Figure } 11 \text { for the Brain LV bootstrap ratios }\end{array}$ \\
\hline & MSEn & & permutation & {$[0.0000,0.0078]$} \\
\hline & & & bootstrap & $\begin{array}{l}\text { Figure } 9 \text { for Task LV confidence intervals and } \\
\text { Figure } 11 \text { for the Brain LV bootstrap ratios }\end{array}$ \\
\hline & MLZ & & permutation & {$[0.0548,0.0865]$} \\
\hline & & & bootstrap & $\begin{array}{l}\text { Figure } 9 \text { for Task LV confidence intervals and } \\
\text { Figure } 11 \text { for the Brain LV bootstrap ratios }\end{array}$ \\
\hline & MLZn & & permutation & {$[0.0018,0.0120]$} \\
\hline & & & bootstrap & $\begin{array}{l}\text { Figure } 9 \text { for Task LV confidence intervals and } \\
\text { Figure } 11 \text { for the Brain LV bootstrap ratios }\end{array}$ \\
\hline
\end{tabular}

normalization were set to 1 for $\mathrm{Y}$-Rest, $\mathrm{Y}$-OnC, and $\mathrm{Y}-\mathrm{OC}$, and to -1 for $\mathrm{O}-\mathrm{R}, \mathrm{O}-\mathrm{OnC}$, and $\mathrm{O}-\mathrm{OC}$, i.e., the main group effect $(Y-O)$. Similarly, the weights for the second contrast were set to 1 for $\mathrm{Y}-\mathrm{R}$ and $\mathrm{O}$-Rest, 0 for $\mathrm{Y}$-OnC, and $\mathrm{O}-\mathrm{OnC}$, and -1 for $\mathrm{Y}-\mathrm{OC}$ and $\mathrm{O}-\mathrm{OC}$, i.e., the main effect of conditions that orders them from the task requiring the least attention and effort (Rest) to the one demanding the most (OC). Our choices for these contrasts were hypoth- eses driven, and as such, they have clear interpretations. However, they were also justified to a large degree in terms of the amount of variance in our data that they actually explain. We confirmed this by running an alternative explorative version of task PLS, namely a "meancentering task PLS". Following this version of the method, not only the brain latent variables but also the task ones are allowed to "rotate" during the SVD of the mean- 

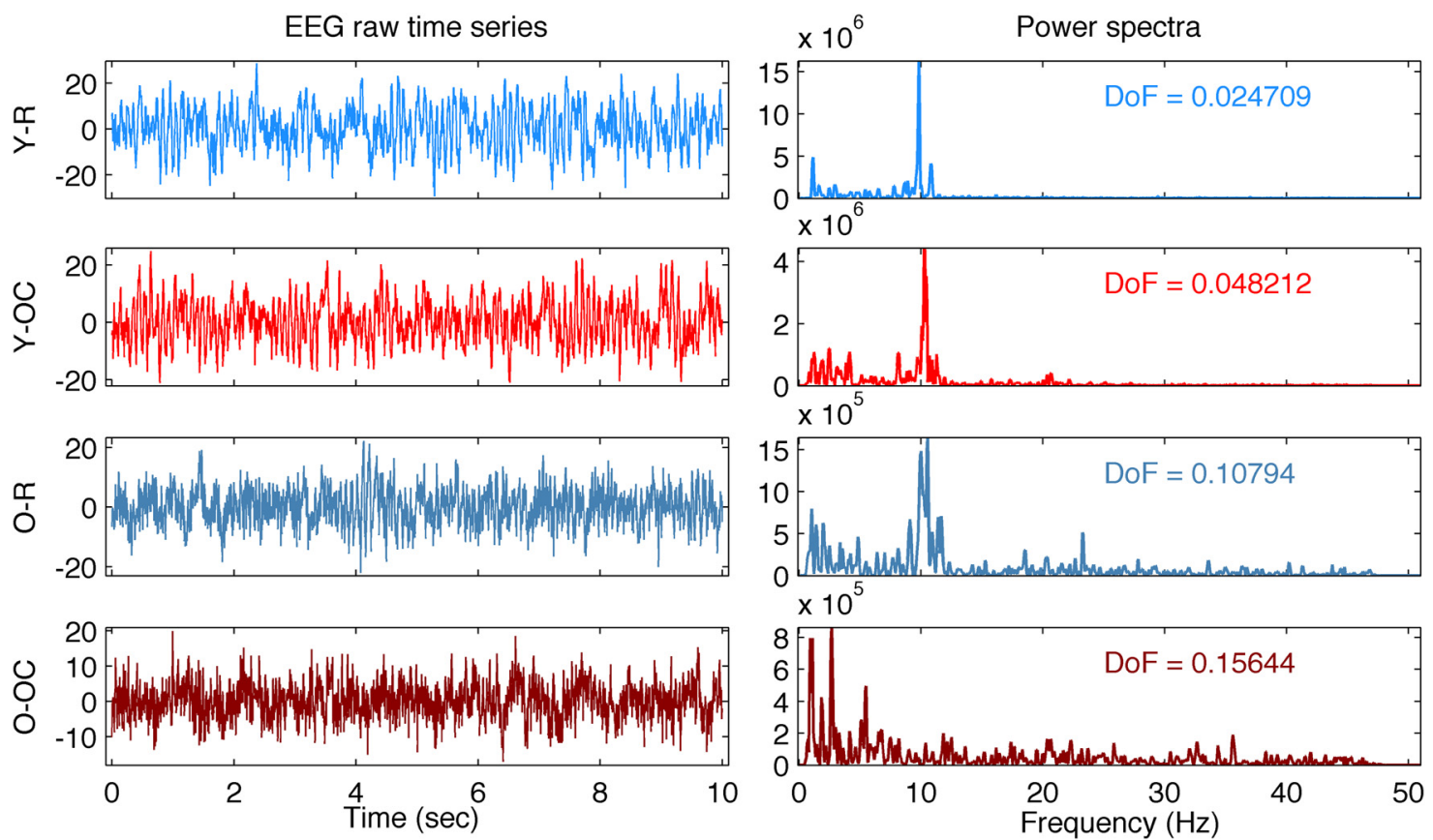

Figure 1 EEG time series and power spectra of randomly chosen data segments. Time series (left column) and power spectra (right column) of randomly chosen data segments for channel $\mathrm{Cz}$ of two participants, one young and one old, are shown. $\mathrm{R}$ condition is presented in in blueish colors, and $\mathrm{OC}$ in reddish colors. From top to bottom, the two conditions for the young participant, and then similarly for the old one. The corresponding DoF is reported with each power spectrum. A peak close to $10 \mathrm{~Hz}$ is apparent in all cases but for the OC condition of the old participant.

centered and concatenated auto-covariance matrix of the initial group data matrices, to explain as much variance of the data as possible (always under the constraint of orthogonality; Mclntosh and Lobaugh, 2004 shows a detailed description of the method). For all metrics, the first two latent variables of the mean-centering task PLS corresponded to contrasts similar (albeit not identical) to the ones we tested (group and condition main effects), and explained approximately $77-99 \%$ and $1-15 \%$ of the total variance, respectively, and $88-99 \%$ in sum.

\section{Results}

To give the reader an intuition on the metrics and their comparability, as well as some guidance in the interpretation of the results, we illustrate in Figures 1 and 2 representative EEG traces and their respective metrics curves. Figure 1, left column, depicts randomly selected data segments from two participants, one young and one old, for the resting state, requiring the least attention, and the Oddball counting, requiring the most attention, conditions. In Figure 1, right column, the corresponding power spectra $(P)$ and the associated DoF are shown. The results of the respective mutliscale metrics are presented in Figure 2. In the following sections, we report the observed effects with respect to aging and experimental conditions for all the different metrics.

\section{Between group differences: aging effects}

We first investigated group differences between young and old participants by performing a separate contrast task PLS analysis for each metric for the main effect $Y-O$.
Group differences can be inspected in Figures 3 and 4, where the mean values with SE intervals are depicted. The $\mathrm{Cz}$ electrode was chosen to visualize mean differences because oddball responses are well represented by the central electrodes (Müller et al., 2008, 2009), and generally $\mathrm{Cz}$ is less affected by muscle artifacts. The permutation tests showed that the contrast was significant for all metrics $(p<0.001$, except for MSE, for which $p=0.002)$. These effects were to a large degree homogeneous among conditions (albeit not identical), and statistically reliable according to the bootstrap tests as shown in Figure 3. In the following we describe the main patterns of the results via the mean and SE intervals (Figs. 3 and 4), and the bootstrap ratios of the brain latent variables (Figs. $6-8$ ). As regards the magnitude of variability metrics (Figs. 3 and 6), it can be seen that the young participants had reliably more power $(\mathrm{P})$ at frequencies below $12 \mathrm{~Hz}$ (with the exception of a narrow band around $8 \mathrm{~Hz}$ ), as well as a larger magnitude of detrended fluctuations $[\ln (F)]$, variance $[\ln (V)]$, and SD. The effects of the last three metrics were generally reliable across channels and scales, although they were the strongest for the parieto-occipital channels and longer time scales. As for the metrics that evaluate the structure of EEG variability across time scales (Figs. 4, 7 and 8), the elderly's degrees of freedom of all channels' power spectra were larger than that of the young participants, i.e., the former's spectra were flatter. Moreover, the DoFs were the highest for the anterior channels, as well as for the lateral ones, which were also noisier (Fig. 4). Figures 4 and 7 also show that the mag- 

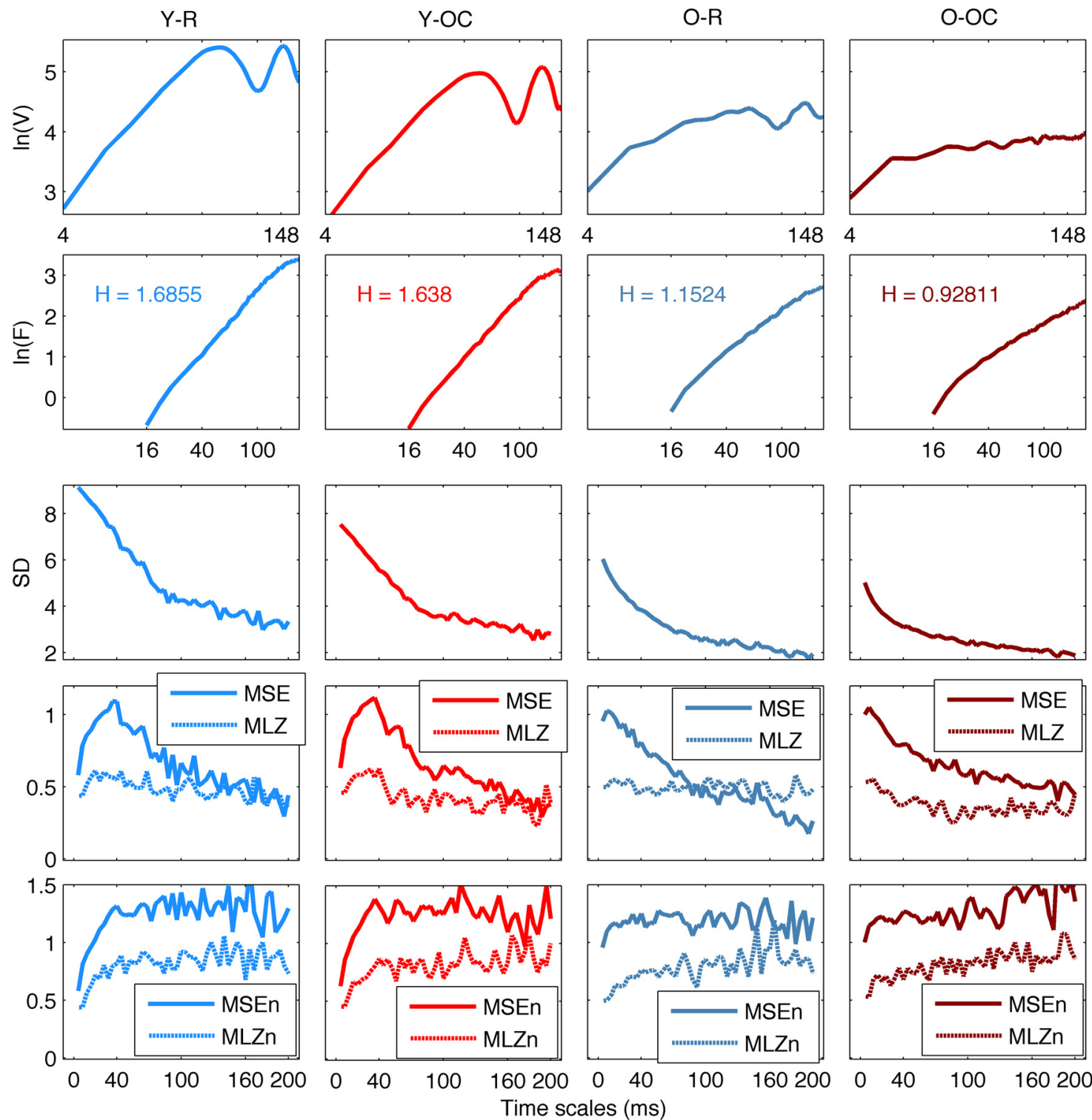

Figure 2 Multiscale metrics of randomly chosen data segments. The multiscale metrics of the same data segments of Figure 1 are shown, the metrics being arranged from top to bottom $\ln (V)$ and $\ln (F)$, also depicting the value of $H$, in logarithmic scale, then, SD, MSE (solid line), and MLZ (dotted line), and finally, SEn (solid line) and MLZn (dotted line), in linear scale, and data segments arranged from left to right column, in the same colors as in Figure 1 . The frequency peaks close to $10 \mathrm{~Hz}$ correspond to local minima of In( $V$ ) at the time scale of $100 \mathrm{~ms}$. The peaks of MSE and MLZ, as well as the first peaks of the $\ln (\mathrm{V})$, close to the time scale of $40 \mathrm{~ms}$, are related to the fact that most of the power of the signals lies below $50 \mathrm{~Hz}$. Accordingly, the instances where $\ln (V)$ reduces again after the time scale of $148 \mathrm{~ms}$ correspond to additional power peaks in the low theta and delta frequencies. However, in general, there is no straightforward relationship between frequencies of the power spectra and time scales of the metrics that undergo either detrending $\ln (F)$ of DFA or coarse graining (SD, MSE, MLZ, MSEn, and MLZn).

nitude of the detrended fluctuations coincided with a larger Hurst exponent for young participants than for the older. On average, $H$ was around 1.5 for older participants and 1.7 for the young one (Fig. 3). In both groups, $H$ values were the highest for more posterior, as well as midline (and also less noisy), channels. With respect to the entropic metrics (Fig. 8), relative to the young participants, entropy was higher for the older participants at time scales shorter than $24 \mathrm{~ms}$, and lower at longer scales from this point on. Exemplified in Figure 4, the MSE curves of channel $\mathrm{Cz}$ across all conditions show a crossing point. The effect below the crossing point (i.e., higher entropy for the old participants for short time scales) was slightly stronger at the parieto-occipital channels, whereas the effect above the crossing point (i.e., higher entropy for the young participants for long time scales) was stronger at the frontocentral channels, and was present at least up to the scale of $80 \mathrm{~ms}$ (Fig. 8). After normalizing for the SD at each scale after coarse graining, the resulting MSEn also showed group differences, but in this case mainly so for 

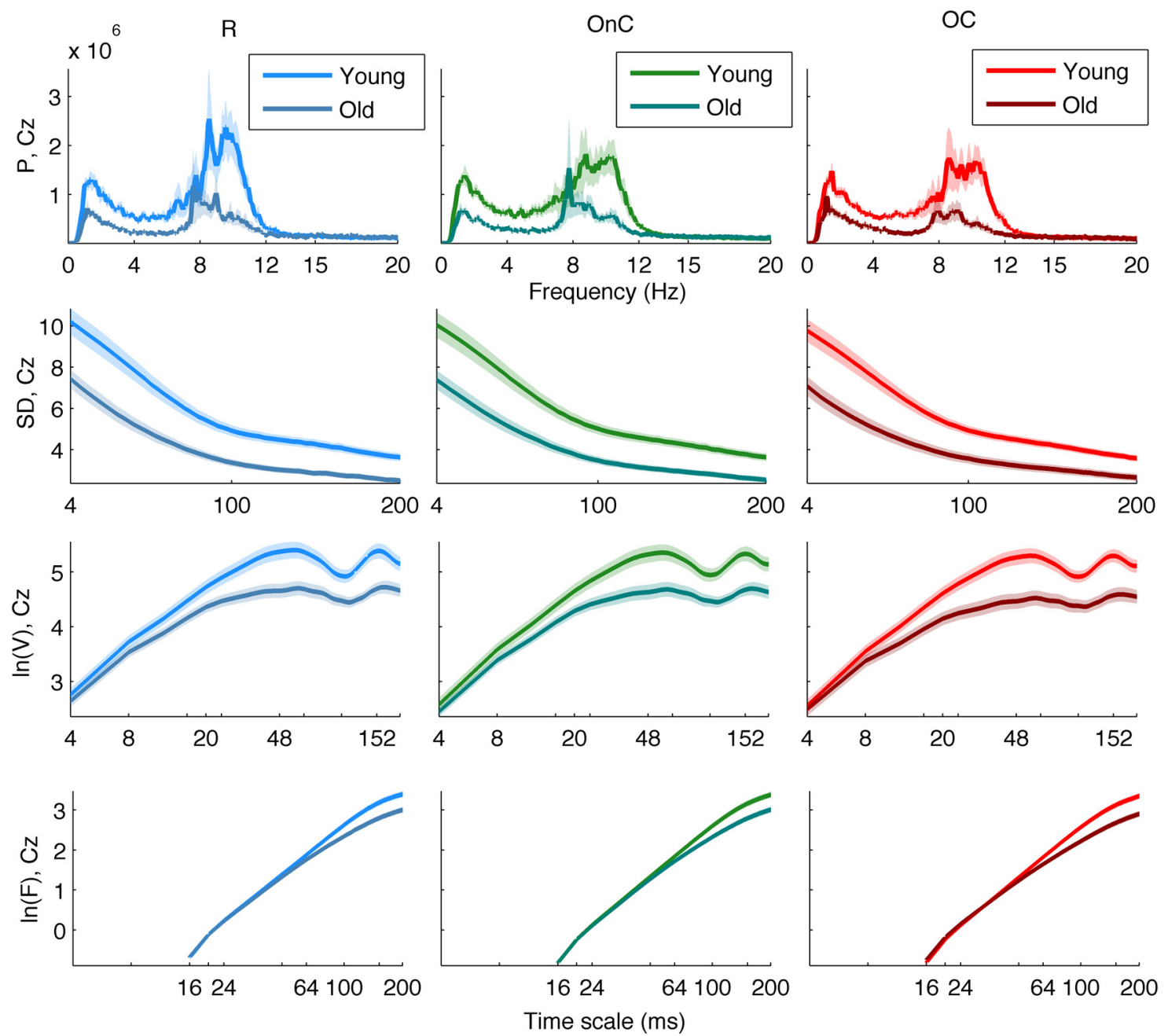

Figure 3 Group means and SE intervals of the metrics of the variability magnitude across conditions. From top to bottom: $P$, $\ln (V), S D$, and $\ln (F)$ are shown for channel $\mathrm{Cz}$, for all conditions (R, bluish colors; OnC, greenish colors; OC, reddish colors, from left to right columns), with darker colors for old participants (lighter for young). Thick lines and areas of faded colors represent the means and the SE intervals, respectively. Horizontal axes depict frequency for $\mathrm{P}$, and time scale logarithmically for $\ln (F)$ and $\ln (V)$, and linearly for SD. Please note the group differences, which are similar (but not identical) among conditions. The magnitude of variability is generally higher for young participants than old participants across scales, particularly so for longer time scales and lower frequencies (except for a small interval $\sim 8 \mathrm{~Hz}$ ).

time scales lower than $32 \mathrm{~ms}$, where SampEn was higher for old participants. In contrast, the differences between groups for longer time scales were not as strong. Results were similar for the Lempel-Ziv entropy metrics (MLZ and MLZn) shown in Figures 4 and 8 . However, effects were statistically weaker than for MSE, and the crossing point tended to be one scale shorter for $M L Z$, i.e., at $20 \mathrm{~ms}$, and one scale longer for MLZn, i.e., at 36 ms.

In summary, the metrics that primarily evaluate the magnitude of variability across scales (the power spectrum, the detrended fluctuations' amplitude, the variogram, and the $\mathrm{SD}$ ), indicated that the young participants exhibited larger fluctuations, mainly so for low-frequencies, long time scales, and for the posterior channels. Inversely, entropy differences between groups reversed at the scale of $20-24 \mathrm{~ms}$, and showed higher entropy for old (young) participants at shorter (longer) time scales, mainly so for posterior (anterior) channels, respectively. Normalizing for the standard deviation after coarse graining substantially weakened the effect at the long time scales. The generalized Hurst exponent, as a metric of complexity (or structure in the variability), was in accordance with the SampEn at long-scales, which was higher for the young participants, whereas the more DoF of the old participants was to be expected given their "flatter" power spectrum, especially for the lower frequencies $<12 \mathrm{~Hz}$.

\section{Effects of experimental conditions}

We next tested for the main effect of condition, mainly contrasting $\mathrm{R}$ and $\mathrm{OC}$, as the $\mathrm{OnC}$ was placed in the middle. The permutation test showed that the contrast was significant with $p<0.001$ for $P, \ln (V), \mathrm{SD}, \ln (F)$, and MSE, and with $p=0.002$ for MSEn, $p=0.069$ for MLZ, and $p=0.005$ for MLZn. The contrast was not significant for DoF and $H(p>0.1)$. Notably, the contrast for condition explained much less variance in our data than that for group, which was revealed by comparing the singular 
$\mathrm{R}$

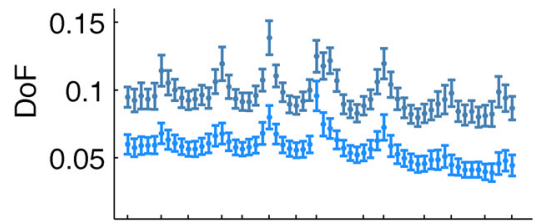

Fp1 Fz FCz Cz CP2 P2 PO4
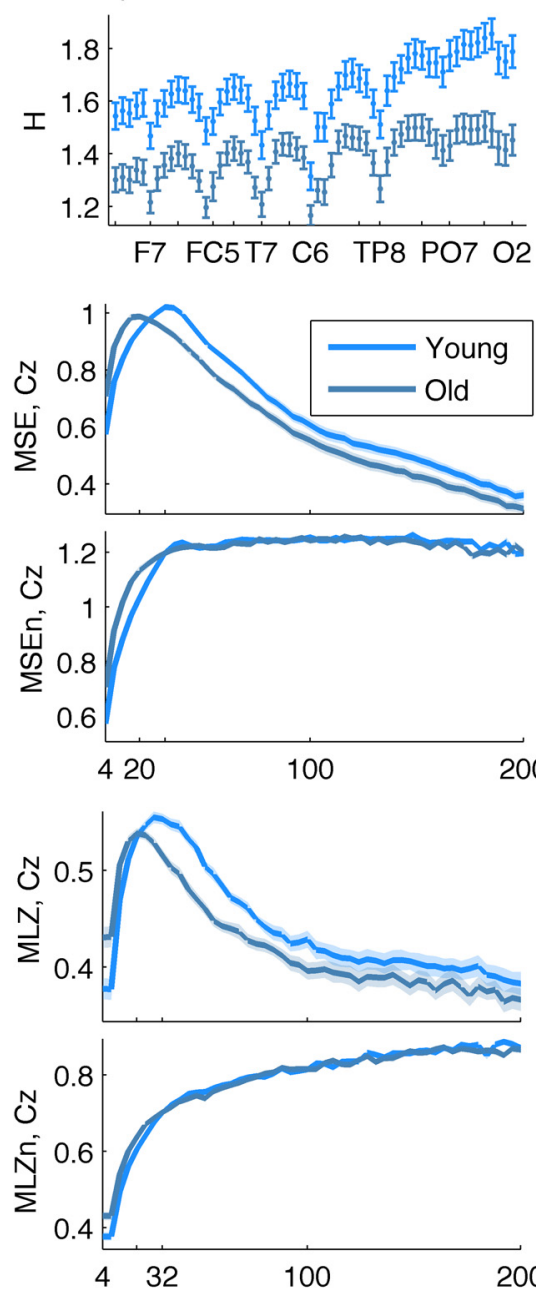

OnC
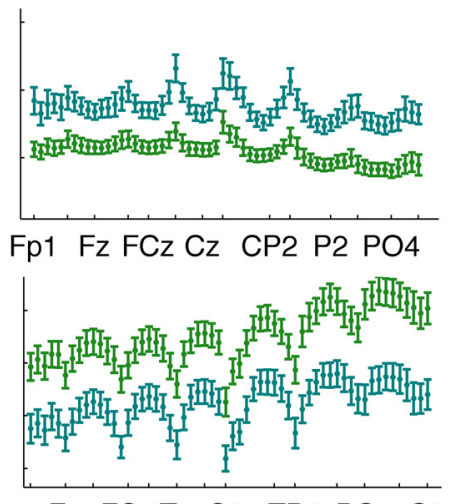

F7 FC5T7 C6 TP8 PO7 O2 Channels
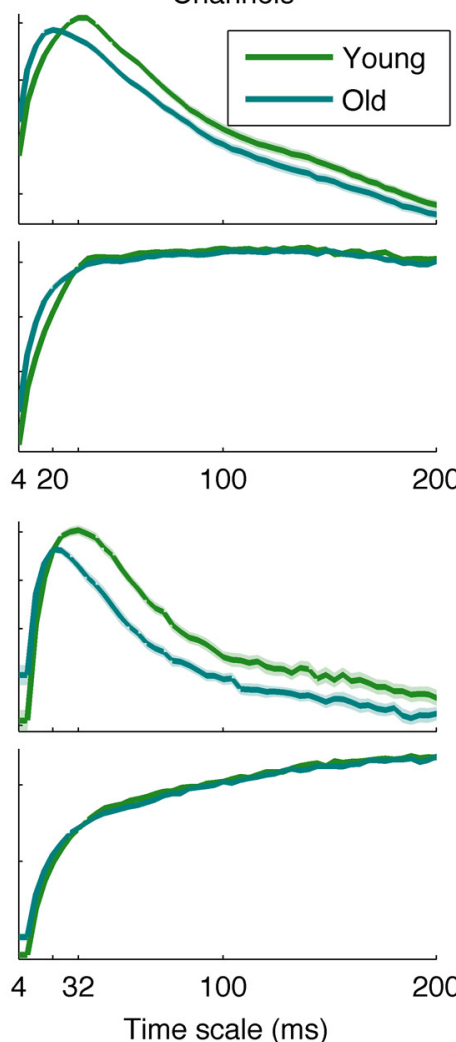

OC

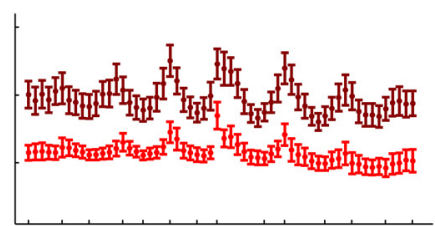

Fp1 Fz FCz Cz CP2 P2 PO4
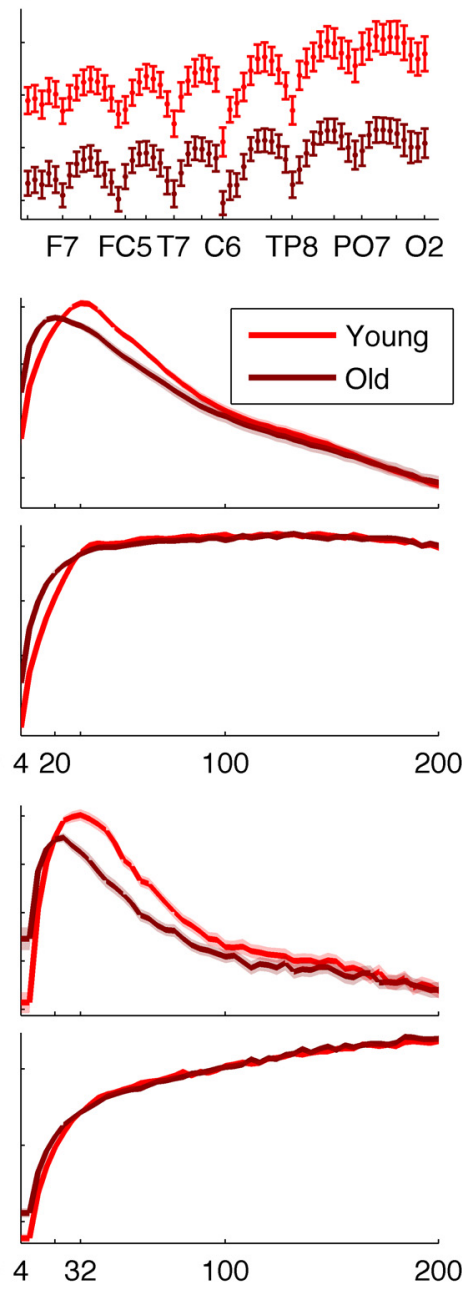

Figure 4 Group means and SE intervals of the metrics of the of variability structure across conditions. From top to bottom: DoF, generalized $H$, MSE, MSEn, MLZ, and MLZn for all conditions. The arrangement of columns, as well as the color and line conventions, are similar to Figure 1, except for DoF and $H$, where error bars are used to depict the standard error intervals. For DoF and $H$, all channels are shown along the horizontal axis (from frontal to occipital and left to right hemisphere ones), whereas channel $\mathrm{Cz}$ is shown for the rest of the metrics. Thus, the horizontal axes for those metrics depict time scale in a linear scale. Please note the group differences, which are similar (but not identical) among conditions. In particular, DoF are more and $H$ is lower for the old participants than the young participants across all channels, MSE and ML $Z$ are higher for old participants for short time scales, $<24$ and $20 \mathrm{~ms}$, respectively, and the inverse for longer scales. MSEn and MLZn are also higher for old participants for scales $<32 \mathrm{~ms}$, but the effect for longer time scales is weaker.

values of the condition contrast for each metric in Figure 8 with the corresponding ones for the group contrast in Figure 5 (the latter were much larger). As further illustrated in Figure 8 , the bootstrap test showed that for $P, \ln (V), S D$, and $\ln (F)$ the three conditions could not be separated reliably with a confidence of $95 \%$ (the respective confidence intervals around the weights of the task latent variables were largely overlapping). Instead, for the en- tropic metrics (MSE, MSEn, MLZ, and MLZn) R was generally reliably separated from the task conditions (OC and OnC), which was more clearly so for young participants. To evaluate the statistically reliable effects, as well as the statistically un- or less-reliable tendencies, we here present the brain latent variables for all metrics (Figs. 10 and 11). As for the metrics of the magnitude of variability, $\ln (V)$ and $\ln (F)$ were generally higher for the resting condition 
$P: p<0.001, s=93125224.0$

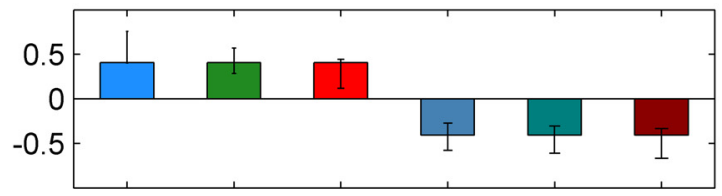

SD: $p<0.001, s=116.4$

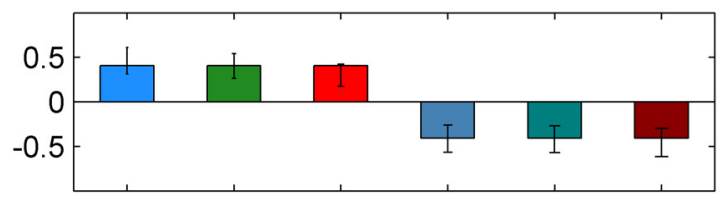

DoF: $p<0.001, s=0.4$

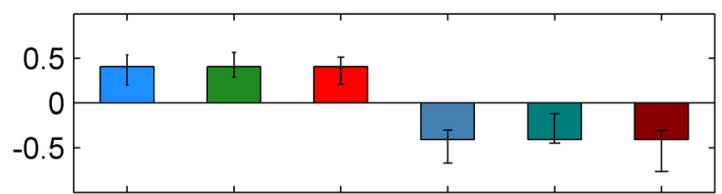

MSE: $p=0.002, s=4.1$

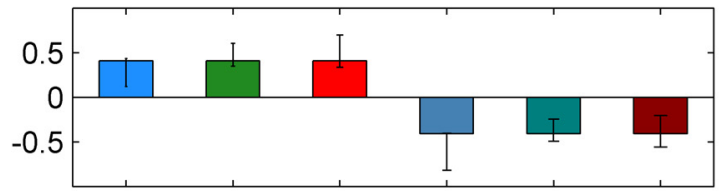

MLZ: $p<0.001, s=1.6$

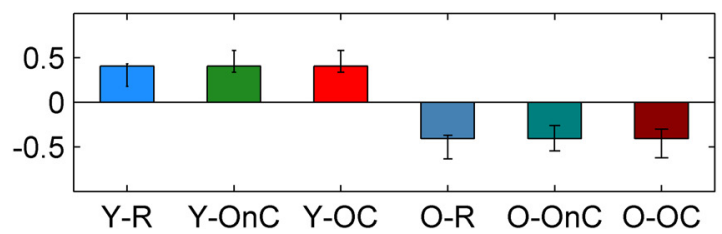

$\ln (V): p<0.001, s=43.6$

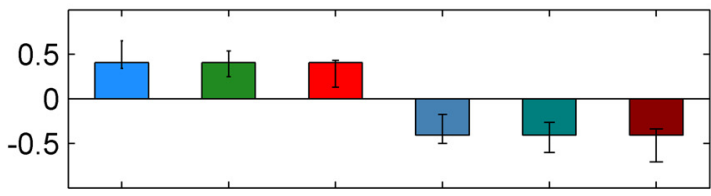

$\ln (F): p<0.001, s=22.8$

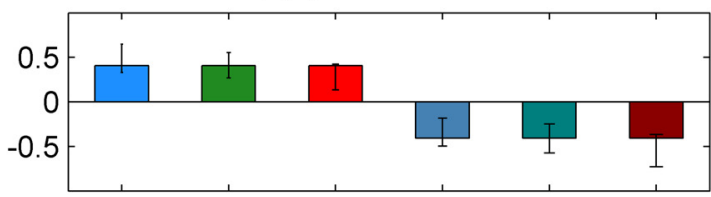

$H: p<0.001, s=2.6$

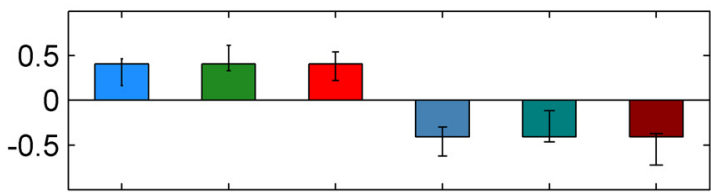

MSEn: $p<0.001, s=3.0$

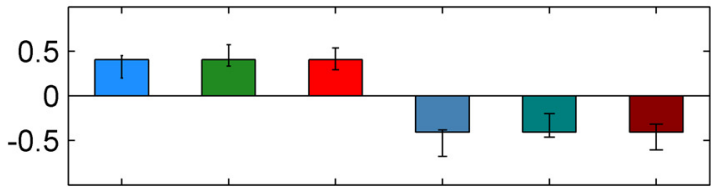

MLZn: $p<0.001, s=1.2$

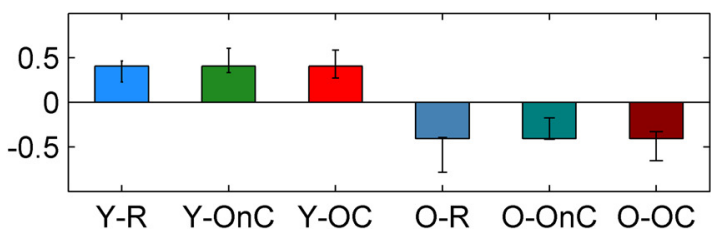

Figure 5 Task latent variables for the group main effect. Each panel shows the weights of the task latent variables of the contrast that corresponds to the group main effect $\mathrm{Y}-\mathrm{O}$. Each bar corresponds to a group-condition combination, with groups being arranged in increasing age from left to right, and conditions arranged in an order of increasing attention and/or task demands (i.e., from R to OC), also from left to right. Color conventions are identical to previous figures. The name of each metric together with the corresponding $p$ value (as derived from the parametric test for significance) and the singular value $s$ of the SVD (proportional to the variance explained by the contrast) are shown on top of the respective panel. Nonoverlapping confidence intervals signify that conditions and/or groups are separated reliably by the contrast. Thus, the contrast is significant and reliably separates the two groups.

across all scales and channels, but particularly so for parieto-occipital channels. The SD was higher also for $\mathrm{R}$ at time scales up to $100 \mathrm{~ms}$, also particularly for the posterior channels. Regarding $P, \mathrm{R}$ had more power in the 5-10 and $15-30 \mathrm{~Hz}$ frequency intervals than the task conditions, whereas OC had more power in the delta band (i.e., $1-4 \mathrm{~Hz}$ ), particularly so for frontocentral channels. Regarding the entropic metrics shown in Figure 11, MSE was higher (lower) for $\mathrm{R}$ than for OC for time scales shorter (longer) than 44-48 ms, respectively. The effect below those scales was stronger for the frontocentral channels. The result for MLZ was very similar for short scales, but was statistically weaker, and with the crossing point moving at shorter scales (32-36 ms). However, above the crossing point, i.e., for long time scales, the effect was practically lost. In addition, MSEn and MLZn were generally higher for $\mathrm{R}$ across all scales, mainly so for the frontocentral channels. This effect was statistically much stronger for the time scales $<56 \mathrm{~ms}$, and more generally for MSEn compared with MLZn.

In summary, the resting state resulted generally in larger fluctuations (except for the SD at long time scales and power at the delta band at the frontal channels). Moreover, the resting condition exhibited higher (lower) entropy than the task condition with counting $(\mathrm{OC})$ at short (long) time scales, respectively. However, after normalizing for the SD at each scale after coarse graining, this effect tended to reverse for long time scales. It is worth noticing that the patterns of results for $P$ and MSE, as well as for MSEn, MLZ, and MLZn, at short scales only for the last three, were to a large degree inverse to those of the group main effect, i.e., the results for the attentive task (rest) condition followed the ones for the young (old) participants. This rough correspondence, however, reversed for the rest of the metrics, i.e., $\ln (V), S D$, and $\ln (F)$. 


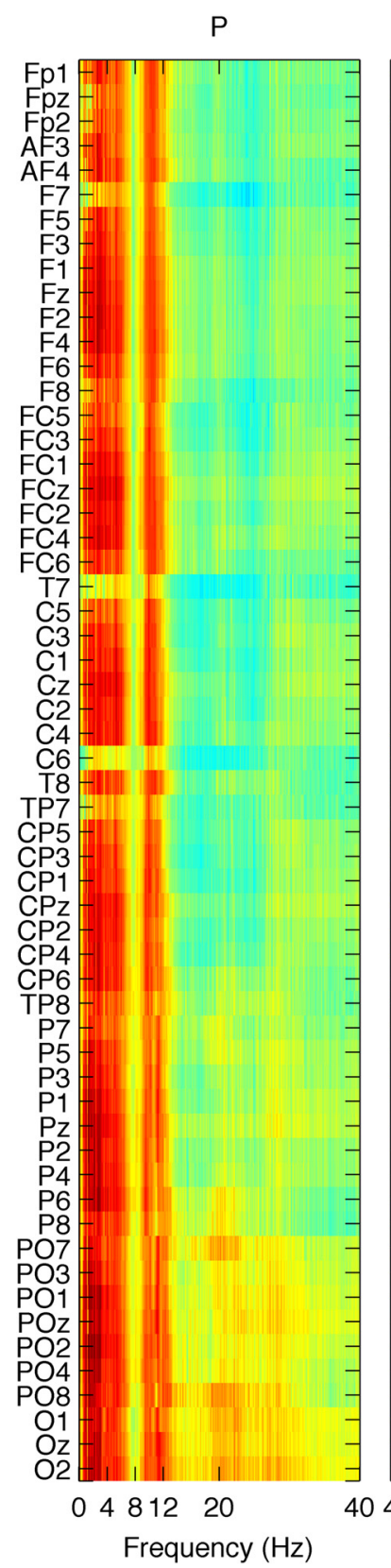

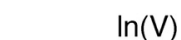
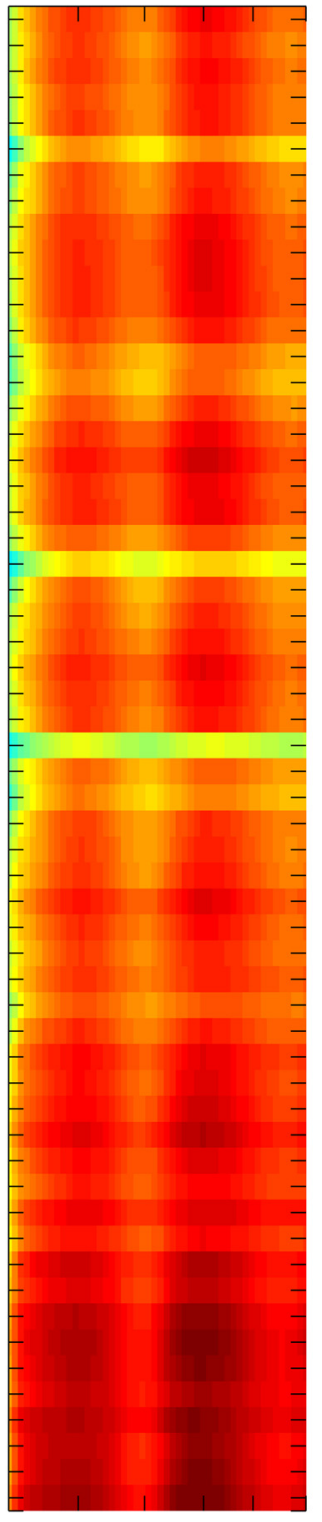

4

Frequency $(\mathrm{Hz}$
SD

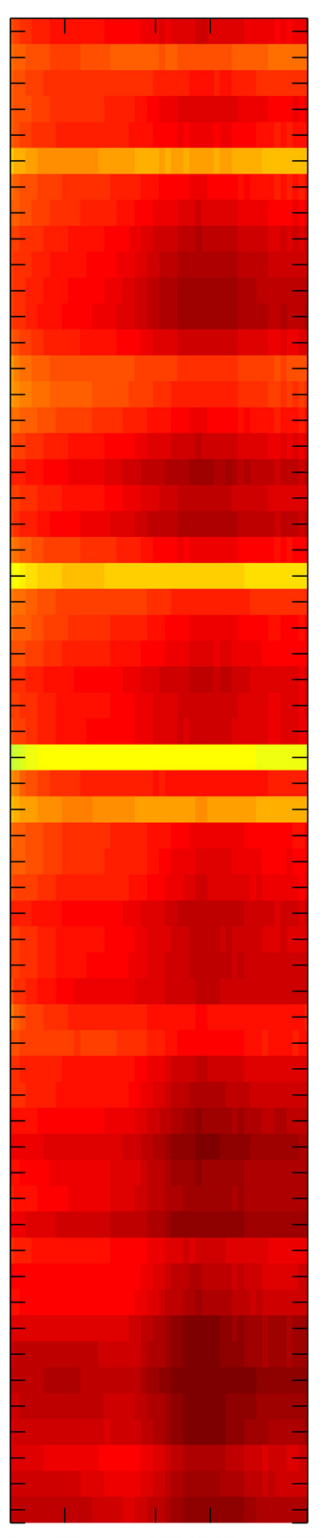

Time scale (ms)
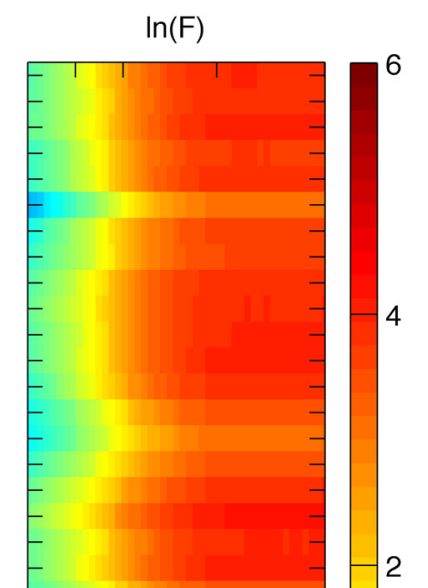

2

$-2$

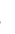

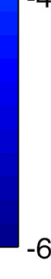

200

6

Figure 6 Brain latent variables for the group main effect of the magnitude of variability metrics. The panels show how much each data element, i.e., a metric's data point, covaries with the contrast that corresponds to the group main effect (Figure 3), in terms of bootstrap ratios, from left to right: $\ln (V), S D$, and $\ln (F)$. Absolute values $>2.5758$ approximate the $99^{\text {th }}$ two-tailed percentile. The vertical axis for all panels depicts channels arranged from top to bottom, starting from frontal and left hemisphere channels, to occipital and right hemisphere ones. The horizontal axes depict frequency for $P$ and time scale in a $\log$ arithmic scale for $\ln (V)$ and $\ln (F)$, and in a linear one for SD. Because the contrast is $\mathrm{Y}-\mathrm{O}$, positive values in reddish colors signify points where young (old) participants had higher values, and the inverse for negative/bluish values. All metrics are higher for young participants: $P$ for frequencies $<12 \mathrm{~Hz}$ with the exception of a short band around $8 \mathrm{~Hz}, \ln (F)$ for time scales longer than 20-32 ms, In( $V)$ for almost all time scales, and SD for all time scales. In general, the effect is statistically stronger for parieto-occipital channels, and for longer time scales and lower frequencies.

\section{Discussion}

The present study investigates the changes of cortical dynamics with aging through the use of a battery of multiscale metrics, which allows that characterize the structure and the magnitude of EEG fluctuations.

\section{Age-related differences in the magnitude of EEG signals variability across time scales}

Our results show that the cortical activity of older participants displayed smaller fluctuations than young participants in a (close to) scale-independent manner. Consistent with 

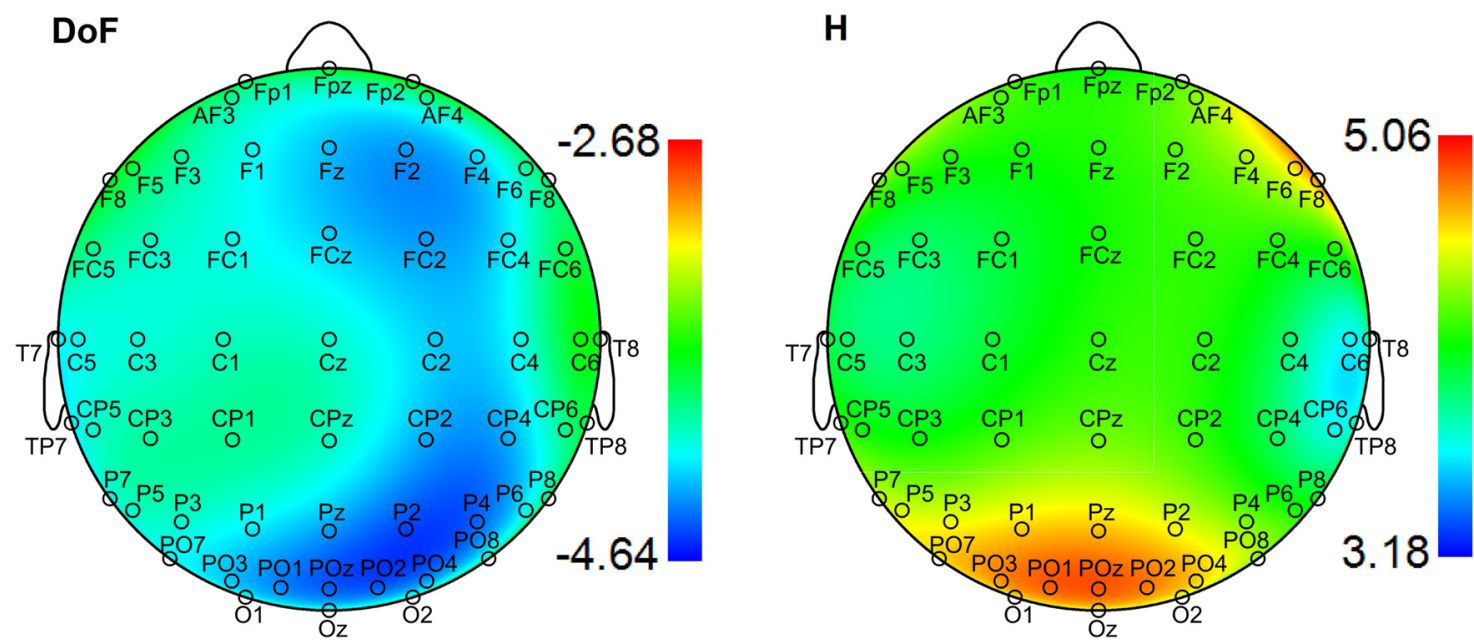

Figure 7 Brain latent variables for groups' main effect for DoF and $H$. The two panels depict the bootstrap ratios of the group main effect for DoF and $H$ (left and right, respectively) across a whole brain with the nose at the top. Interpretations are the same as in Figure 4. The old participants showed reliably more DoF and lower $H$ for (almost) all channels than the young participants.

previous studies (Dustman et al., 1993, 1999; Gaal et al., 2010; Müller and Lindenberger, 2012), EEG signals of the elderly generally contained less spectral power than that of the young adults. Similarly, the DFA, SD, and variogram results also indicated a decrease in the fluctuations' magnitude with aging. Although, to our knowledge, this aspect of brain signal variability has never been explicitly addressed before in EEG recordings, it is in-line with recent fMRI studies (Garrett et al., 2013a,b), where older adults were found to display a reduction of SD BOLD signals in most brain areas (especially cortical) in both resting and task-driven states (Garrett et al., 2011, 2013a). Our study extends these observations to scalp EEG and shows that it is indeed a pervasive characteristic of the aging brain across time scales.

\section{Effects of aging on the organization of cortical fluctuations across time scales}

In the frequency domain, older adults showed flatter power spectra with a lower alpha peak, and more spectral DoF, suggestive of increased 'broadband' noisiness of the cortical activity. Further, long-range autocorrelations were less present in older participants' data (higher $H$ exponent). The multiscale entropy metrics revealed a time-scale-dependence of aging effects regardless of the used estimator (SampEn or LZ) with the elderly's EEG signals being more irregular at fine/shorter scales, and less complex at coarser/longer scales. Thus, young and old brains appear to operate at different time constants making them, under the effect of coarse graining, reach maximal entropy at different time scales. After reaching their respective peak, both young and older adults' MSE/ MLZ curves decreased; however, those of the young remained significantly higher. This loss of complexity across the long scales may be indicative of diminished global information integration with aging, because these scales relate mostly to low-frequency oscillations mediating long-range interactions. Mind, however, that the inverse does not directly apply, because the short scales enclose information about both high- and low-frequency oscillations. Furthermore, it is known also that (multiscale) entropy-based measures reflect both variance and correlation properties of time series (Costa et al., 2002, 2005). To extract variance-related changes, we compared the multiscale entropy curves (MSE, MLZ) with their normalized versions (MSEn, MLZn) and the SD curves. A crossing-over was present for the entropy metrics (regardless of the normalization), but not for SD, for which young and elderly's curves were parallel. It is notable however, that, although the age-group differences in entropy remained mostly significant after normalization, they were substantially weakened. The normalization affected essentially the part of MSE/MLZ curves after the peak that contains the scales accounting for the autocorrelated (low frequencies) content of the signal, which actually contain the most power $(\sim<20 \mathrm{~Hz})$.

The above results are in accordance with the current literature, and extend it with several new findings. First, we reproduced McIntosh et al.'s (2014) results and extended them to longer time scales, as well as to resting-state activity. For the first time scale, our findings (i.e., more irregularity for older adults) are consistent with those of other EEG studies using single-scale measures of complexity (Anokhin et al. 1996; Pierce et al. 2000, 2003; Müller and Lindenberger 2012). Conversely, our observations at longer scales approximate the observations of $\mathrm{fMRI}$ studies, in which the time resolution is much lower than in EEG. Indeed, fMRI investigations at resting state have also shown a loss of entropy with aging (Yang et al., 2013; Smith et al., 2014; Sokunbi, 2014).

Overall, we show that aging effects on cortical fluctuations are time-scale-dependent with regard to structure (i.e., less regular fluctuations at shorter scales and less complex fluctuations at longer scales), but not in terms of magnitude (i.e., a systematic reduction regardless of the time scale). 


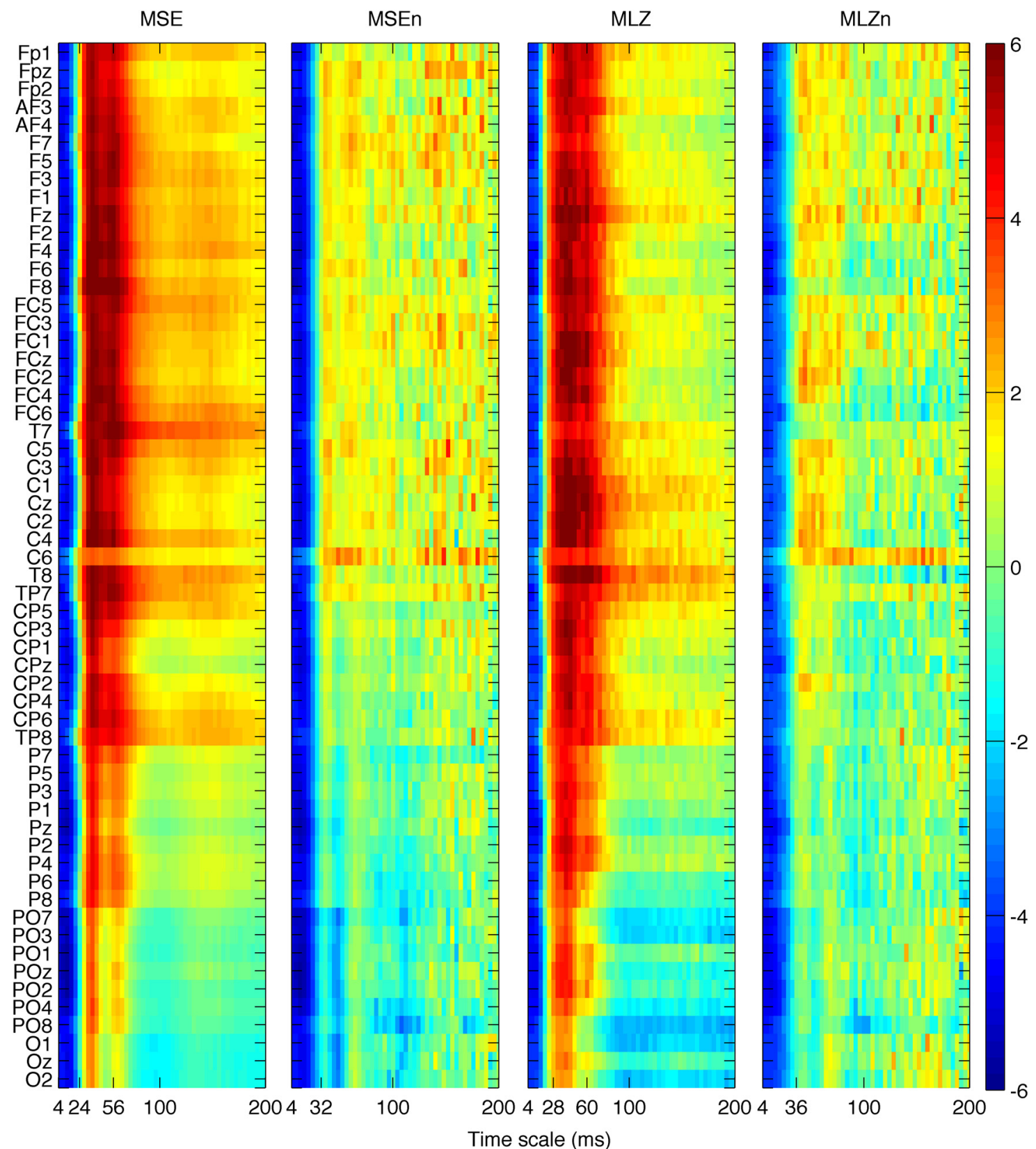

Figure 8 Brain latent variables for groups' main effect of metrics of the structure of variability. The panels depict the bootstrap ratios of the group main effect for MSE, MSEn, MLZ, and MLZn, from left to right. Interpretations, vertical axes, and color conventions are the same as in Figure 4. The horizontal axes depict time scale in a linear scale. All metrics are higher for old participants below some scale (approximately 24, 32, 20, and $36 \mathrm{~ms}$, respectively); this effect is statistically stronger for parieto-occipital channels. Above these scales, MSE and MLZ are higher for young participants than for the old participants up to at least the scale of $80 \mathrm{~ms}$. This effect is marginally reliable for MSEn and MLZn, and stronger for frontocentral channels.

\section{Spatial patterns of variability changes with aging}

The observed aging effects on EEG variability were rather robust for almost all channels. Nevertheless, some spatial patterns showing stronger effects for certain electrodes were distinguishable over the scalp. Notably, the posterior channels were found to display the highest young-old differences in terms of variability magnitude (across all scales), with the younger adults being furthermore variable than the elderly for these regions. This was also the case for the power spectrum, the variogram, and the DFA analyses. This spatial pattern of age-related differences in terms of fluctuations magnitude is consistent with the observation that young adults have more power in most of the frequency bands at the posterior areas (seen in our results, and previously reported by Gaal et al., 2010). Conversely, entropy-wise, young-old differences for the longer/coarser scales were stronger at the frontocentral channels, because EEG signals of the younger partici- 
P: $p<0.001, s=26402522.0$

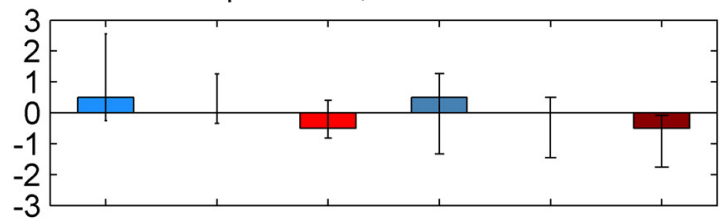

SD: $p<0.001, s=15.3$

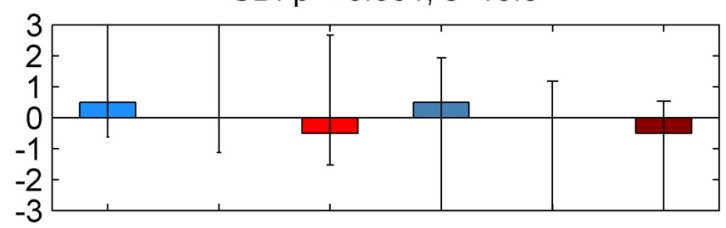

MSE: $p<0.001, s=2.2$

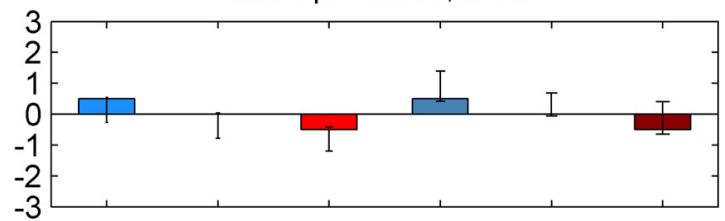

MLZ: $p=0.069, s=0.4$

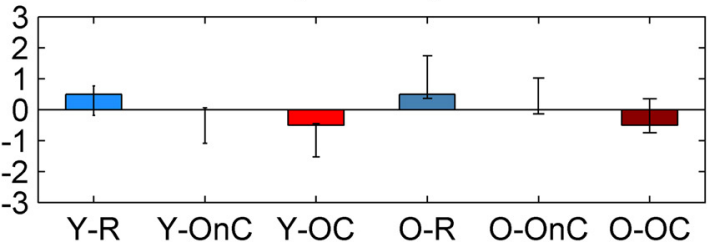

$\ln (V): p<0.001, s=9.8$

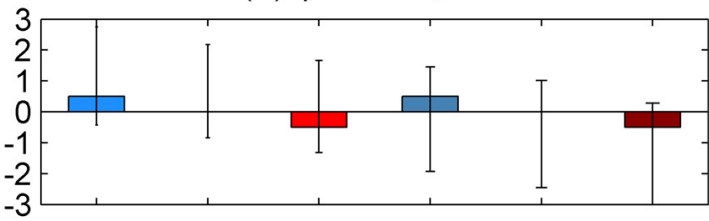

$\ln (F): p<0.001, s=5.9$

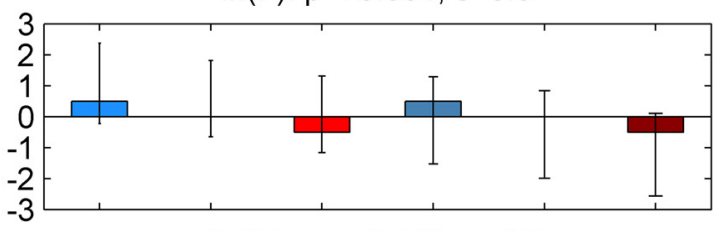

MSEn: $\mathrm{p}=0.002, \mathrm{~s}=0.7$

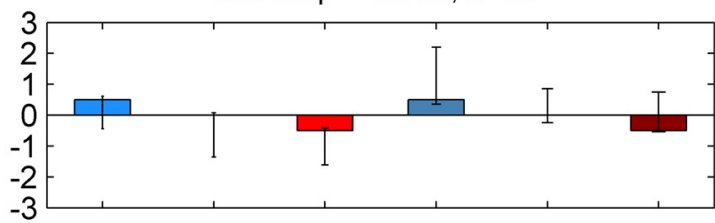

MLZn: $p=0.005, s=0.4$

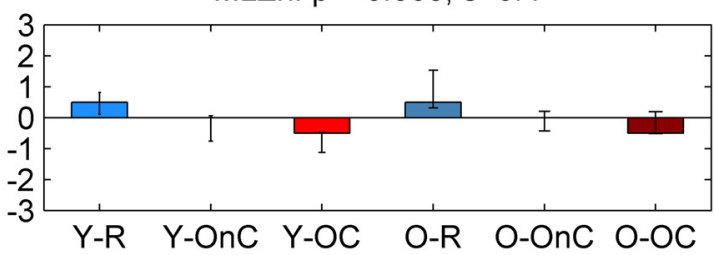

Figure 9 Task latent variables for condition main effect. This figure has an identical arrangement and conventions as Figure 3 (DoF and $H$ are omitted because they were not significant). This latent variable contrasts $\mathrm{R}$ versus OdC with OnC being in the middle, i.e., it arranges conditions in an order of increasing attention and/or task demands. It is significant for almost all metrics with a $p<0.001$, except for MSEn and MLZn that have slightly higher values ( $p=0.002$ and $p=0.005$, respectively), whereas MLZ is significant only to a value of $p=0.069$. However, confidence intervals are largely overlapping, i.e., conditions are not separated reliably, except for the entropic measures (MSE, MSEn, MLZ, and MLZn), where R is generally separated reliably from the task conditions, mainly so for young participants.

pants were more complex for these channels than for the occipital ones.

These results suggest that the detected anterioposterior difference in the magnitude of group effects stems from a fronto-occipital differentiation expressed only in the younger adults' brains. This interpretation corroborates the view of spatial dedifferentiation in the aged brain, as shown for instance in Garrett et al.'s (2013a,b) studies, wherein older adults were found to exhibit low and nearly indistinguishable levels of variability across brain structures in both resting and task-driven states.

\section{Differences between experimental conditions}

The differences between resting and the auditory stimuli conditions (with and without counting) followed a similar pattern across metrics, with the contrast driven mainly by the difference between resting state and the cognitively most demanding oddball counting task. However, this distinction could only be made reliably through MSE, and more consistently so for young participants. The limited change between rest and task situations might be related to the fact that in all experimental conditions participants were instructed to keep their eyes closed. Eyes opening was indeed shown to significantly affect brain signals complexity elsewhere (Hogan et al., 2012; Müller and Lindenberger, 2012), as was also found in our preliminary analysis including the eyes-open condition. In addition, the cognitive task we used is not very demanding. With respect to MSE, the pattern of difference between the resting (least demanding) and oddball counting (most demanding) condition resembled the one differentiating the age groups (old vs young): the EEG of the less demanding task was more complex at shorter scales. A stronger difference was found for the frontocentral channels, most likely due to the attentional load imposed by the task. This difference was reversed at longer scales, where the OdC condition yielded the most entropic signals. To our best knowledge, this is the first time a specific MSE pattern with obvious time-scale-dependence is shown to differentiate between brain states at different cognitive loads. Nevertheless, the low differentiability between conditions in elderly was reported previously and seems to be one of the general signatures characterizing the senescent brain (Garrett et al. 2013a). This lack of specificity in the aged brain manifests itself, thus, both through a spatial (within experimental condition, as shown in the section before) and a "states" (between conditions) dedifferentiation. 


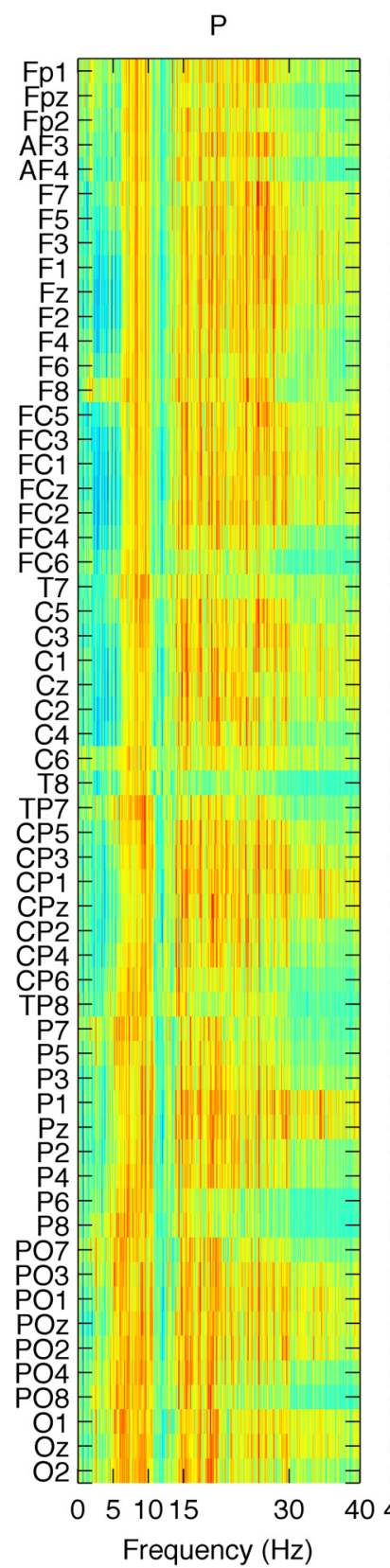

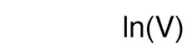
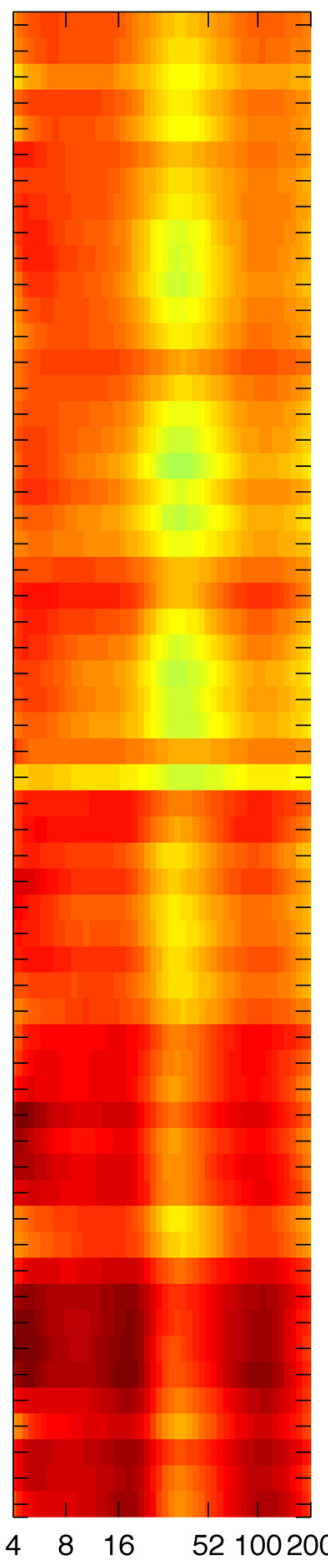

521002004

equency $(\mathrm{Hz})$
SD
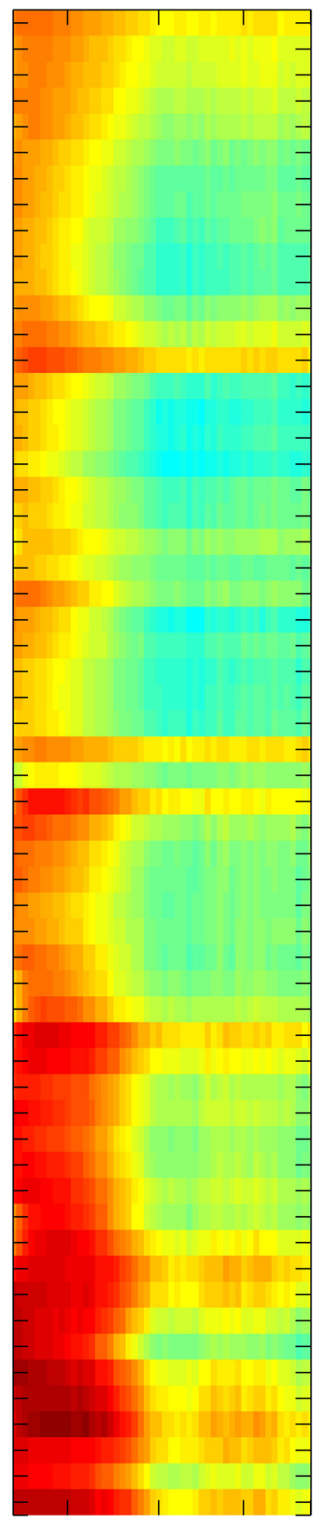

$\begin{array}{lll}40 & 100 & 156 \\ \text { Time scale }(\mathrm{ms})\end{array}$

$\ln (\mathrm{F})$
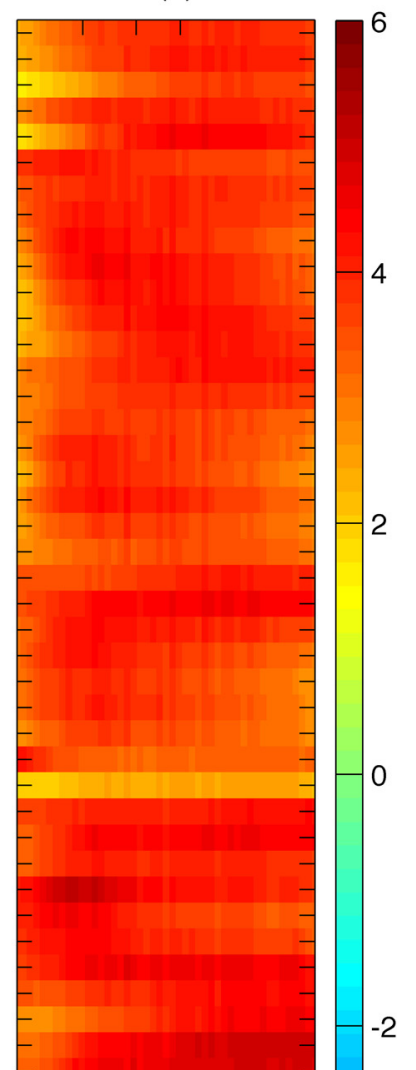

Figure 10 Brain latent variables for the condition main effect of the variability magnitude metrics. The panels depict the bootstrap ratios of the condition main effect for $P, \ln (V), S D$, and $\ln (F)$, from left to right. Interpretations, axes, and color conventions are the same as in Figure 4, only now positive (negative) values in reddish (bluish) colors signify values that were higher for condition $\mathrm{R}(\mathrm{OC})$. In( $V$ ) and $\ln (F)$, were generally higher for the resting condition across all scales and channels, but mainly so for parieto-occipital channels. $\mathrm{SD}$ was higher also for $\mathrm{R}$ for shorter time scales up to $100 \mathrm{~ms}$, also mainly for posterior channels. Regarding $P$, R had more power in the 5-10 and 15-30 Hz frequency intervals, whereas OC had more power in the delta band, mainly so for frontocentral channels. Notice that $P$ has almost an inverse pattern with the groups' main effect in Figure 4.

\section{Convergence of aging theories and empirical findings}

The dedifferentiation hypothesis initially introduced by Baltes and Lindenberger (1997) is repeatedly referred to in the literature to describe and explain cognitive declines with advanced age (for review, see Park and ReuterLorenz, 2009). Notwithstanding its initial framework (i.e., correlations between sensory and cognitive functions), dedifferentiation can be used to account for several facets of age-related changes in brain and behavior (SleimenMalkoun et al., 2014). In the brain, it can be seen through increased interdependence between functional domains (e.g., cognition and motor control; Schäfer et al., 2006, 2010), decreased specialization of brain regions (Park et al., 2004; Dennis and Cabeza, 2011), and more widespread activations (Reuter-Lorenz et al., 2000; Heuninckx 


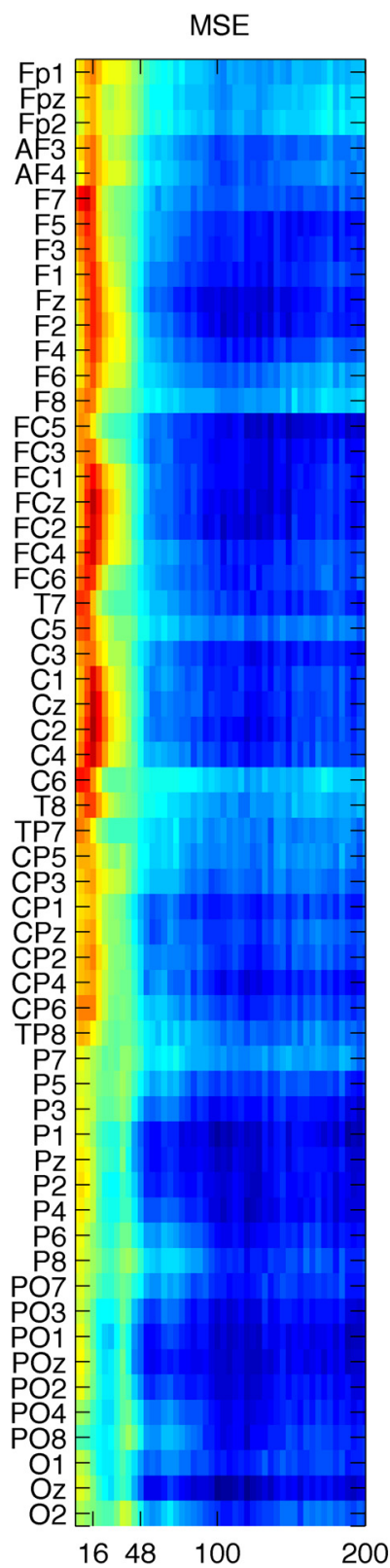

\section{MSEn}

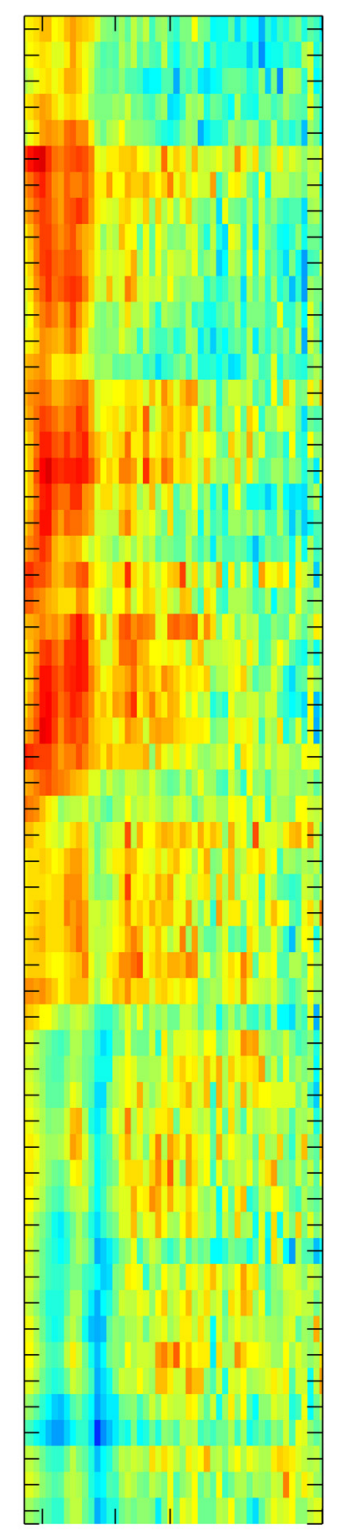

2001

64100
MLZ
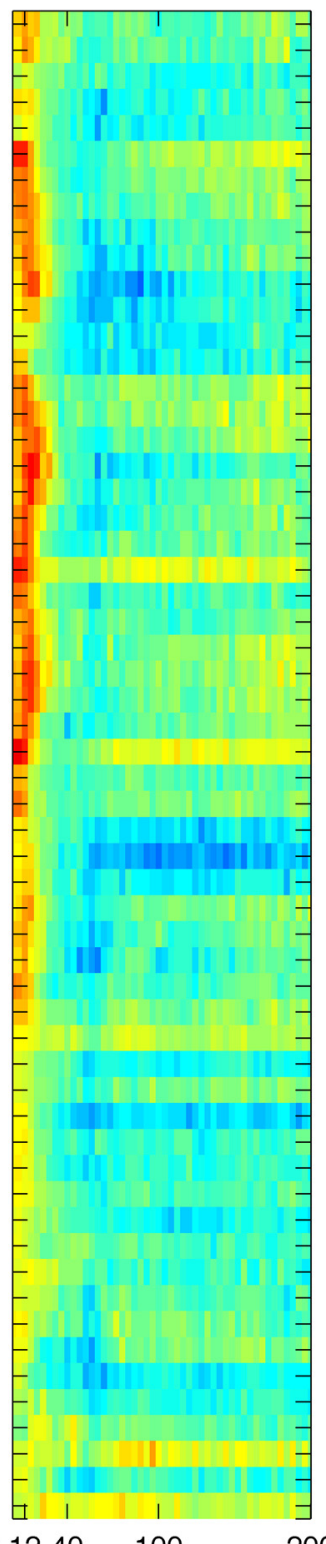

Time scale (ms)

Figure 11 Brain latent variables for conditions' main effect for the structure of variability metrics. The panels depict the bootstrap ratios of the condition main effect for MSE, MSEn, MLZ, and MLZn from left to right. Interpretations, axes, and color conventions are the same as in Figure 6, only now positive values in reddish colors signify values that were higher for condition $\mathrm{R}$ (OC), and the inverse for negative (bluish) values. All metrics are higher for $\mathrm{R}$ below some scale $(\sim 48,56,36$, and 56 ms, respectively) for frontocentral channels. MSE is higher for OC for scales above $48 \mathrm{~ms}$ for all channels, as well, whereas MSEn and MLZn showed a statistically weaker tendency to be higher for $\mathrm{R}$ for scales further than the points mentioned above and for almost all channels. Notice that the pattern of the results is to a large degree inverse to the results of the group main effect, mainly so for MSE and for short scales.

et al., 2005, 2008). Nevertheless, neurobehavioral variability is not an outcome measure in the dedifferentiation approach and its extensions. In this regard, for a long time, the aging literature essentially focused on behavioral variability (e.g., response times) in relation to changes in patterns of brain activations (Hultsch et al., 2008; MacDonald et al., 2009), rather than on characterizing brain signals fluctuations themselves. The neural noise hypoth- esis (Li et al., 2000; Li and Sikström, 2002) is one of the first and most established approaches dealing with this aspect. It argues in favor of an increased random background activity in the aged CNS (referred to as neural noise), resulting in a higher intraindividual variability in performance (Li et al., 2000, 2001; Li and Sikström, 2002; Hultsch et al., 2002). Currently, it is widely recognized that the variability of brain activations in space and time is of 
high relevance to understand brain functioning in health (e.g., development; Vakorin et al., 2011; and normal aging; Mclntosh et al., 2014) and disease (e.g., autism; Bosl et al., 2011; and Alzheimer disease; Mizuno et al., 2010). This rather recent interest succeeds a more established view in the domains of physiology and motor behavior where the loss of complexity hypothesis $(\mathrm{LOCH})$ was developed (Lipsitz and Goldberger, 1992; Lipsitz, 2002, 2004). In this framework, the structure of fluctuations is considered to reflect the complexity of the underlying functional organization and interactions within and between different subsystems. The $\mathrm{LOCH}$ stipulates that during aging, as well as disease, there is a generic tendency toward less-complex (behavioral and physiological) outputs that could be in the direction of an increased regularity or an increased randomness (Goldberger, 1996; Vaillancourt and Newell, 2002, 2003), both supposedly indicative of a breakdown of functional synergies and a decoupling of components. The LOCH can be connected to dedifferentiation of brain activations by looking at the spatial distribution of variability and linking time scales of fluctuations to information processing in the brain. A more uniform spatial representation of variability across cortical and subcortical structures expresses the characteristic spatial dedifferentiation of the aging brain (Garrett et al., 2011). Conversely, the time scales view presumes that complexity at finer scales characterizes local processing, and may thus be related to short neural connections, whereas the coarser scales (by filtering out higher frequencies) reflect the more long-range (i.e., global) interactions, and therefore depend on longer neuronal fibers (Mizuno et al., 2010; Vakorin et al., 2011; Mclntosh et al. 2014). Mclntosh et al. (2014) argued in favor of this assumption and showed that scale differences observed with MSE follow closely those that can be quantified through other entropy measures that distinguish local and distributed informational exchanges (i.e., conditional entropy and mutual information).

All the aforementioned aging hypotheses could be linked to underlying alterations of neural structures and interactions, as well as dysregulation of neurotransmission, together leading to a less rich and flexible repertoire of functional synergies. Structurally and physiologically, the aging brain is known to incur changes characterized by a marginal neuronal loss (Wickelgren, 1996; Morrison and Hof, 1997; Bishop et al., 2010), but a substantial decline in the integrity of white matter (Sullivan et al., 2010; Madden et al., 2012), as well as a disruption in the synthesis of some neuro-transmitters (dopamine, norepinephrine, acetylcholine). These modifications greatly affect large-scale brain networks by disturbing interhemispheric functional connections and interactions (Duffy et al., 1996; Kikuchi et al., 2002; Langan et al., 2010), as well as somatosensory cortical inhibition (Cheng and Lin, 2013). Impaired dopaminergic neurotransmission further compromises the modulation of neural noise, which is an additional cause of inflexibility of brain activity and behavior (Hong and Rebec, 2012). The conjunction of all these alteration is most likely responsible for the observed changes in multiscale variability and activation patterns, which nicely merges with the predictions stemming from main aging theories. Indeed, although these theories were developed to cover different domains and mechanisms, they converge to describe systemic modifications characterizing the senescence process(es) in the neurobehavioral system (Sleimen-Malkoun et al., 2014). An essential current debate that needs to be settled is the relative importance of local (i.e., grey matter and neurotransmission degradation) and global (i.e., white matter degradation and demyelination) network changes, as well as the beneficial or detrimental role of stochastic components of brain dynamics (i.e., noise), and how these factors affect functional connectivity, brain signal variability, and performance. In the framework of dedifferentiation the degradation of neurotransmission is thought to reduce the signal-to-noise ratio in local networks leading to less distinct cortical representations, and potentially to less specific functional connectivity ( $\mathrm{Li}$ and Lindenberger, 1999; Li et al. 2001; Li and Sikström, 2002). Functional connectivity and complexity are considered to entertain an inverse relationship, according to which higher entropy is found when connectivity is poor, and vice versa (Friston, 1996; Müller and Lindenberger, 2012; Ghanbari et al., 2013). However, from a different perspective, the reverse is commonly suggested (cf. Vakorin et al., 2011) based on the assumption that information processing and (neural) complexity go hand in hand (Tononi et al., 1994, 1998; Slifkin and Newell, 1999). Conversely, following the finding that neural information transmission is determined by both the degree and time scale of synchrony (Baptista and Kurths, 2008), a different view can be suggested. Accordingly, neural processing would be maximized when synchronization is high at coarse time scales (strong connectivity requiring complexity to be low) and low at fine scales (weak connectivity allowing the expression of greater complexity). Evidence for such time-scale-dependence, with a negative connectivity-complexity association at fine scales and the reverse at coarser scales was found in resting-state fMRI data (McDonough and Nashiro, 2014), as well as in mean field model and BOLD simulations (Jirsa et al. 2010; Nakagawa et al., 2013). Therefore, it could be concluded that entropic and variability changes convey different information depending on the time scale under scrutiny. More precision should be gained in the future by accounting for the recently uncovered nonstationarity of the dynamics of resting state fMRI (Allen et al., 2014), which is expressed through different functional connectivity measures for different time windows and moments in time. Hansen et al. (2015) demonstrated that the nonstationarity of the resting-state dynamics is evident in rapid changes in functional connectivity patterns, which are otherwise relatively invariant during epochs lasting one to two minutes. These transitions are reminiscent of phase transitions as known from statistical physics and were referred to as functional connectivity dynamics (FCD; Hansen et al., 2015). A successful quantification of FCD promises to provide a more profound understanding of variability- and complexity-related phenomena in brain networks, and thus ageing-related changes in brain and behavior. 
Overall, it appears that although the aging brain displays more widespread activations, in terms of information processing, it is characterized by an increased spatial clustering with a shift toward a lesser contribution of long-range connections (Meunier et al., 2009). However, the contribution of changes in connectivity and nonstationaries remains to be unraveled.

\section{Conclusion}

Our findings provide support to the importance of multiscale brain signal variability as a means to assess the effects of aging on brain functioning. Even though no absolute value or a single metric can currently be offered as a biomarker of brain age, the contribution of a systematic study of variability through multiple measures and scales rests in the link that can be established with functional and structural connectivity, as well as the richness of activation patterns. Nevertheless, we argue that any expected or discussed effect of aging should meet the complexity of the functional organization within the human neurophysiological and neurobehavioral system, which makes simple, strict, and irrevocably generalizable correspondences unlikely to be found. It would be misleading, for instance, to expect that aging is a process of "loss", and that what is observed in term of behavior mirrors sensu stricto changes in brain activations. In the brain, what counts most to insure a rich adaptable behavior is the interplay between multiple factors, namely, local and global neuroanatomical connectivity, noise levels, and interaction delays (Ghosh et al., 2008; Jirsa et al., 2010; Deco et al., 2011). Accordingly, the healthy brain expresses critical magnitudes and structures of variability that undergo significant changes with development, aging, and disease. Regarding aging, some general features can be extracted. Mainly, a pervasive reduced level of variability, in terms of magnitude, an increased irregularity at shorter time scales, a decrease complexity at long scales, and finally a spatial dedifferentiation in activations and between brain states (e.g., rest vs task). The meaning of these changes and their link with structure, function, and dynamics can be significantly furthered and made more explicit through theoretical and simulation studies, and empirical investigations. Systematic investigation of how aging-relevant functional and structural modifications affect the outcome of multiscale variability and complexity metrics would offer a major contribution. A wider set of entropy estimators (e.g., epsilon entropy) and metrics can also be covered (multivariate measures, synchronization measures, Lyapunov exponents, etc.). However, it is to be expected that these supplementary methods will provide converging evidence in terms of global effects, as it has been found in the present study for the measures quantifying fluctuations' magnitude and those quantifying their structure. Therefore, based on our findings we contend that adding more metrics would not profoundly advance our current understanding of aging. Conversely, a novel and more promising direction would be appropriately taking into account the nonstationary nature of brain processes, which seem to be an inherent property of brain functioning and to occur on various scales of organization
(Hansen et al., 2015). Finally, combining different modalities of brain imaging and investigating different brain states in a single aging experiment would make it possible to irrefutably relate the different phenomena that have been separately shown to characterize aging (e.g., dedifferentiation, loss of complexity, variability changes), as well as integrate newly uncovered ones (e.g., nonstationaries in functional connectivity; Allen et al., 2014; Hansen et al., 2015), while establishing the link with performance and behavior.

\section{References}

Adler RL, Weiss B (1967) Entropy: a complete metric invariant for automorphisms of the torus. Proc Natl Acad Sci U S A 57:15731576. Medline

Allen EA, Damaraju E, Plis SM, Erhardt EB, Eichele T, Calhoun VD (2014) Tracking whole-brain connectivity dynamics in the resting state. Cereb Cortex 24:663-76. CrossRef Medline

Anokhin AP, Birbaumer N, Lutzenberger W, Nikolaev A, Vogel F (1996) Age increases brain complexity. Electroencephalogr Clin Neurophysiol 99:63-68. Medline

Baltes PB, Lindenberger U (1997) Emergence of a powerful connection between sensory and cognitive functions across the adult life span: a new window to the study of cognitive aging? Psychol Aging 12:12-21. Medline

Baptista MS, Kurths J (2008) Transmission of information in active networks. Phys Rev E Stat Nonlin Soft Matter Phys 77:026205. Medline

Bishop NA, Lu T, Yankner BA (2010) Neural mechanisms of ageing and cognitive decline. Nature 464:529-535. CrossRef Medline

Bosl W, Tierney A, Tager-Flusberg H, Nelson C (2011) EEG complexity as a biomarker for autism spectrum disorder risk. BMC Med 9:18. CrossRef Medline

Bravi A, Longtin A, Seely A (2011) Review and classification of variability analysis techniques with clinical applications. BioMed Eng OnLine 10:90. CrossRef Medline

Brown LD, Cai TT, DasGupta A (2001) Interval estimation for a binomial proportion. Statist Sci 16:101-133. CrossRef

Bryce RM, Sprague KB (2012) Revisiting detrended fluctuation analysis. Sci Rep 2:315. CrossRef Medline

Cheng CH, Lin YY (2013) Aging-related decline in somatosensory inhibition of the human cerebral cortex. Exp Brain Res 226:145152. CrossRef Medline

Conte E, Khrennikov A, Federici A, Zbilut JP (2009) Fractal fluctuations and quantum-like chaos in the brain by analysis of variability of brain waves: a new method based on a fractal variance function and random matrix theory: a link with El Naschie fractal Cantorian space-time and $\mathrm{V}$. Weiss and $\mathrm{H}$. Weiss golden ratio in brain. Chaos Solitons Fract 41:2790-2800. CrossRef

Costa M, Goldberger AL, Peng CK (2002) Multiscale entropy analysis of complex physiologic time series. Phys Rev Lett 89:068102. Medline

Costa M, Goldberger AL, Peng CK (2005) Multiscale entropy analysis of biological signals. Phys Rev E Stat Nonlin Soft Matter Phys 71:021906. Medline

Cressie NAC (1993) Statistics for Spatial Data. New York: Wiley.

Deco G, Jirsa V, McIntosh AR, Sporns O, Kötter R (2009) Key role of coupling, delay, and noise in resting brain fluctuations. Proc Natl Acad Sci USA 106:10302-10307. CrossRef Medline

Deco G, Jirsa VK, Mclntosh AR (2011) Emerging concepts for the dynamical organization of resting-state activity in the brain. Nat Rev Neurosci 12:43-56. CrossRef Medline

Deco G, Jirsa VK, Mclntosh AR (2013) Resting brains never rest: computational insights into potential cognitive architectures. Trends Neurosci 36:268-274. CrossRef Medline 
Dennis NA, Cabeza R (2011) Age-related dedifferentiation of learning systems: an fMRI study of implicit and explicit learning. Neurobiol Aging 32:2318.e17-2330. CrossRef

Duffy FH, Mcanulty GB, Albert MS (1996) Effects of age upon interhemispheric EEG coherence in normal adults. Neurobiol Aging 17:587-599. Medline

Dustman RE, Shearer DE, Emmerson RY (1993) EEG and eventrelated potentials in normal aging. Prog Neurobiol 41:369-401. Medline

Dustman RE, Shearer DE, Emmerson RY (1999) Life-span changes in EEG spectral amplitude, amplitude variability and mean frequency. Clin Neurophysiol 110:1399-1409. Medline

Faisal AA, Selen LPJ, Wolpert DM (2008) Noise in the nervous system. Nat Rev Neurosci 9:292-303. CrossRef Medline

Feldman DP, Crutchfield JP (1998) Measures of statistical complexity: why? Physics Lett A 238:244-252. CrossRef

Friston KJ (1996) Theoretical neurobiology and schizophrenia. $\mathrm{Br}$ Med Bull 52:644-655. Medline

Gaal ZA, Boha R, Stam CJ, Molnár M (2010) Age-dependent features of EEG-reactivity-spectral complexity, and network characteristics. Neurosci Lett 479:79-84. CrossRef

Gammaitoni L, Hanggi P, Jung P, Marchesoni F (1998) Stochastic resonance. Rev Mod Phys 70:223-287. CrossRef

Garrett D, Kovacevic N, McIntosh AR, Grady C (2011) The importance of being variable. J Neurosci 31:4496-4503. CrossRef Medline

Garrett DD, Kovacevic N, McIntosh AR, Grady CL (2013a) The modulation of BOLD variability between cognitive states varies by age and processing speed. Cereb Cortex 23:684-93. CrossRef Medline

Garrett DD, Samanez-Larkin GR, MacDonald SW, Lindenberger U, McIntosh AR, Grady CL (2013b) Moment-to-moment brain signal variability: a next frontier in human brain mapping? Neurosci Biobehav Rev 37:610-624. CrossRef Medline

Ghanbari Y, Bloy L, Christopher Edgar, J, Blaskey L, Verma R, Roberts TL (2013) Joint analysis of band-specific functional connectivity and signal complexity in autism. J Autism Dev Disord 44:444-460.

Ghosh A, Rho Y, McIntosh AR, Kötter R, Jirsa VK (2008) Noise during rest enables the exploration of the brain's dynamic repertoire. PLoS Comput Biol 4:e1000196. CrossRef Medline

Goldberger AL (1996) Non-linear dynamics for clinicians: chaos theory, fractals, and complexity at the bedside. Lancet 347:13121314. Medline

Grady CL, Garrett DD (2014) Understanding variability in the BOLD signal and why it matters for aging. Brain Imaging Behav 8:274283. [PMC] [10.1007/s11682-013-9253-0] [Medline]

Gratton G, Coles MG, Donchin E (1983) A new method for off-line removal of ocular artifact. Electroencephalogr Clin Neurophysiol 55:468-484. Medline

Hansen EC, Battablia D, Spiegler A, Deco G, Jirsa VK (2015) Functional connectivity dynamics: modeling the switching behavior of the resting state. Neuroimage 105:525-535.

Hardstone R, Poil SS, Schiavone G, Jansen R, Nikulin VV, Mansvelder HD, Linkenkaer-Hansen K (2012) Detrended fluctuation analysis: a scale-free view on neuronal oscillations. Front Physiol 3:450. CrossRef Medline

Heuninckx S, Wenderoth N, Debaere F, Peeters R, Swinnen SP (2005) Neural basis of aging: the penetration of cognition into action control. J Neurosci 25:6787-6796. CrossRef Medline

Heuninckx S, Wenderoth N, Swinnen SP (2008) Systems neuroplasticity in the aging brain: recruiting additional neural resources for successful motor performance in elderly persons. J Neurosci 28: 91-99. CrossRef Medline

Hlaváčková-Schindler K (2009) Causality in time series: its detection and quantification by means of information theory. In: Information theory and statistical learning (Emmert-Streib F, Dehmer M, eds), pp 183-207. New York: Springer.

Hogan MJ, Kilmartin L, Keane M, Collins P, Staff RT, Kaiser J, Lai R, Upton N (2012) Electrophysiological entropy in younger adults, older controls and older cognitively declined adults. Brain Res 1445:1-10. CrossRef Medline

Hong SL, Rebec GV (2012) Biological sources of inflexibility in brain and behavior with aging and neurodegenerative diseases. Front Syst Neurosci 6:77. CrossRef Medline

Hultsch DF, Macdonald SWS, Dixon RA (2002) Variability in reaction time performance of younger and older adults. J Gerontol B Psychol Sci Soc Sci 57:101-115. CrossRef Medline

Hultsch DF, Strauss E, Hunter MA, Macdonald SW (2008) Intraindividual variability, cognition, and aging. In: The handbook of aging and cognition, Ed 3 (Craik FIM, Salthouse TA, eds), pp 491-556. New York: Psychology Press.

Hurst HE (1951) Long-term storage capacity of reservoirs. Trans Amer Soc Civ Eng 116:770-808.

Huys R, Perdikis D, Jirsa VK (2014) Functional architectures and structured flows on manifolds: a dynamical framework for motor behavior. Psychol Rev 121:302-336. CrossRef Medline

Jirsa VK, Sporns O, Breakspear M, Deco G, Mcintosh AR (2010) Towards the virtual brain: network modeling of the intact and the damaged brain. Arch Ital Biol 148:189-205. Medline

Kikuchi M, Wada Y, Takeda T, Oe H, Hashimoto T, Koshino Y (2002) EEG harmonic responses to photic stimulation in normal aging and Alzheimer's disease: differences in interhemispheric coherence. Clin Neurophysiol 113:1045-1051. Medline

Krishnan A, Williams LJ, Mclntosh AR, Abdi H (2011) Partial least squares (PLS) methods for neuroimaging: a tutorial and review. Neuroimage 56:455-475. CrossRef Medline

Langan J, Peltier SJ, Bo J, Fling BW, Welsh RC, Seidler RD (2010) Functional implications of age differences in motor system connectivity. Front Syst Neurosci 4:17. CrossRef Medline

Lempel A, Ziv J (1976) On the complexity of finite sequences. IEEE Trans Information Theory 22:75-81. CrossRef

Lesne A, Blanc JL, Pezard L (2009) Entropy estimation of very short symbolic sequences. Phys Rev E Stat Nonlin Soft Matter Phys 79:046208. Medline

Li S, Lindenberger U (1999) Cognitive neuroscience of memory (Nilsson LG, Markowitsch HJ, eds), pp 103-146. Toronto: Hogrefe \& Huber.

Li S, Lindenberger U, Sikström S (2001) Aging cognition: from neuromodulation to representation. Trends Cogn Sci 5:479-486. Medline

Li SC, Lindenberger U, Frensch PA (2000) Unifying cognitive aging: from neuromodulation to representation to cognition. Neurocomputing 32-33:879-890. CrossRef

Li SC, Sikström S (2002) Integrative neurocomputational perspectives on cognitive aging, neuromodulation, and representation. Neurosci Biobehav Rev 26:795-808. Medline

Lipsitz LA (2002) Dynamics of stability: the physiologic basis of functional health and frailty. J Gerontol A Biol Sci Med Sci 57: B115-B125. Medline

Lipsitz LA (2004) Physiological complexity, aging, and the path to frailty. Sci Aging Knowledge Environ 2004:pe16. CrossRef Medline

Lipsitz LA, Goldberger AL (1992) Loss of complexity and aging: potential applications of fractals and chaos theory to senescence. JAMA 267:1806-1809. Medline

Liu CY, Krishnan AP, Yan L, Smith RX, Kilroy E, Alger JR, Ringman JM, Wang DJ (2013) Complexity and synchronicity of resting state blood oxygenation level-dependent (BOLD) functional MRI in normal aging and cognitive decline. J Magn Reson Imaging 38:36-45. CrossRef

MacDonald SWS, Li SC, Bäckman L (2009) Neural underpinnings of within-person variability in cognitive functioning. Psychol Aging 24:792-808. CrossRef Medline

Madden DJ, Bennett IJ, Burzynska A, Potter GG, Chen NK, Song AW (2012) Diffusion tensor imaging of cerebral white matter integrity in cognitive aging. Biochim Biophys Acta 1822:386-400. CrossRef Medline

McDonnell MD, Abbott D (2009) What is stochastic resonance? Definitions, misconceptions, debates, and its relevance to biology. PLoS Comput Biol 5:e1000348. CrossRef Medline 
McDonnell MD, Ward LM (2011) The benefits of noise in neural systems: bridging theory and experiment. Nat Rev Neurosci 12: 415-426. CrossRef Medline

McDonough IM, Nashiro K (2014) Network complexity as a measure of information processing across resting-state networks: evidence from the Human Connectome Project. Front Hum Neurosci 8:409. CrossRef

Mclntosh AR, Kovacevic N, Lippe S, Garrett D, Grady C, Jirsa V (2010) The development of a noisy brain. Arch Ital Biol 148:323337. Medline

Mclntosh AR, Lobaugh NJ (2004) Partial least squares analysis of neuroimaging data: applications and advances. Neuroimage 23: S250-263. CrossRef

Mclntosh AR, Vakorin V, Kovacevic N, Wang H, Diaconescu A, Protzner AB (2014) Spatiotemporal dependency of age-related changes in brain signal variability. Cereb Cortex 24:1806-1017. CrossRef Medline

Meunier D, Achard S, Morcom A, Bullmore E (2009) Age-related changes in modular organization of human brain functional networks. Neuroimage 44:715-723. CrossRef Medline

Mizuno T, Takahashi T, Cho RY, Kikuchi M, Murata T, Takahashi K, Wada Y (2010) Assessment of EEG dynamical complexity in Alzheimer's disease using multiscale entropy. Clin Neurophysiol 121: 1438-1446. CrossRef Medline

Morrison JH, Hof PR (1997) Life and death of neurons in the aging brain. Science 278:412-419. Medline

Mueller V, Brehmer Y, Von Oertzen T, Li SC, Lindenberger U (2008) Electrophysiological correlates of selective attention: a lifespan comparison. BMC Neurosci 9:18. CrossRef Medline

Müller V, Gruber W, Klimesch W, Lindenberger U (2009) Lifespan differences in cortical dynamics of auditory perception. Dev Sci 12:839-853. CrossRef Medline

Müller V, Lindenberger U (2012) Lifespan differences in nonlinear dynamics during rest and auditory oddball performance. Dev Sci 15:540-556. CrossRef Medline

Nakagawa TT, Jirsa VK, Spiegler A, Mcintosh AR, Deco G (2013) Bottom up modeling of the connectome: linking structure and function in the resting brain and their changes in aging. Neuroimage 80:318-329. CrossRef Medline

Nikulin VV, Brismar T (2004) Comment on "Multiscale entropy analysis of complex physiologic time series". Phys Rev Lett 92: 089803. CrossRef

Ornstein DS, Weiss B (1991) Statistical Properties of chaotic systems. Bull Amer Math Soc 24:11-116. CrossRef

Park DC, Polk TA, Park R, Minear M, Savage A, Smith MR (2004) Aging reduces neural specialization in ventral visual cortex. Proc Natl Acad Sci U S A 101:13091-13095. CrossRef Medline

Park DC, Reuter-Lorenz P (2009) The adaptive brain: aging and neurocognitive scaffolding. Annu Rev Psychol 60:173-196. CrossRef Medline

Peng CK, Buldyrev SV, Havlin S, Simons M, Stanley HE, Goldberger AL (1994) Mosaic organization of DNA nucleotides. Phys Rev E 49:1685-1689. Medline

Pierce TW, Kelly SP, Watson TD, Replogle D, King JS, Pribram KH (2000) Age differences in dynamic measures of EEG. Brain Topogr 13:127-134. Medline

Pierce TW, Watson TD, King JS, Kelly SP, Pribram KH (2003) Age differences in factor analysis of EEG. Brain Topogr 16:19-27. Medline
Pincus SM (1991) Approximate entropy as a measure of system complexity. Proc Natl Acad Sci USA 88:2297-2301. CrossRef

Reuter-Lorenz PA, Jonides J, Smith EE, Hartley A, Miller A, Marshuetz C, Koeppe RA (2000) Age differences in the frontal lateralization of verbal and spatial working memory revealed by PET. J Cogn Neurosci 12:174-187. Medline

Richman JS, Moorman JR (2000) Physiological time-series analysis using approximate entropy and sample entropy. Am J Physiol Heart Circ Physiol 278:H2039-H2049. Medline

Schaefer S, Schumacher V (2010) The interplay between cognitive and motor functioning in healthy older adults: findings from dualtask studies and suggestions for intervention. Gerontology 57: 239-246. CrossRef Medline

Schäfer S, Huxhold O, Lindenberger U (2006) Healthy mind in healthy body? A review of sensorimotor-cognitive interdependencies in old age. Eur Rev Aging Phys Act 3:45-54. CrossRef

Sleimen-Malkoun R, Temprado JJ, Hong SL (2014) Aging induced loss of complexity and dedifferentiation: consequences for coordination dynamics within and between brain, muscular and behavioral levels. Front Aging Neurosci 6:140. CrossRef

Slifkin AB, Newell KM (1999) Noise, information transmission, force variability. J Exp Psychol Hum Percept Perform 25:837-851. Medline

Smith RX, Yan L, Wang DJ (2014) Multiple time scale complexity analysis of resting state FMRI. Brain Imaging Behav 8:284-291. CrossRef Medline

Sokunbi MO (2014) Sample entropy reveals high discriminative power between young and elderly adults in short fMRI data sets. Front Neuroinform 8:69. CrossRef Medline

Sullivan EV, Rohlfing T, Pfefferbaum A (2010) Quantitative fiber tracking of lateral and interhemispheric white matter systems in normal aging: relations to timed performance. Neurobiol Aging 31:464481. CrossRef Medline

Tononi G, Edelman GM, Sporns O (1998) Complexity and coherency: integrating information in the brain. Trends Cogn Sci 2:474-484. Medline

Tononi G, Sporns O, Edelman GM (1994) A measure for brain complexity: relating functional segregation and integration in the nervous system. Proc Natl Acad Sci USA 91:5033-5037. Medline

Vaillancourt DE, Newell KM (2002) Changing complexity in human behavior and physiology through aging and disease. Neurobiol Aging 23:1-11. Medline

Vaillancourt DE, Newell KM (2003) Aging and the time and frequency structure of force output variability. J Appl Physiol 94:903-912. CrossRef Medline

Vakorin VA, Lippé S, Mclntosh AR (2011) Variability of brain signals processed locally transforms into higher connectivity with brain development. J Neurosci 31:6405-6413. CrossRef Medline

Vigário RN (1997) Extraction of ocular artefacts from EEG using independent component analysis. Electroencephalogr Clin Neurophysiol 103:395-404. CrossRef

Wickelgren I (1996) For the cortex, neuron loss may be less than thought. Science 273:48-50. Medline

Yang AC, Huang CC, Yeh HL, Liu ME, Hong CJ, Tu PC, Chen JF, Huang NE, Peng CK, Lin CP, Tsai SJ (2013) Complexity of spontaneous BOLD activity in default mode network is correlated with cognitive function in normal male elderly: a multiscale entropy analysis. Neurobiol Aging 34:428-438. CrossRef Medline 